\title{
Biological phase separation: cell biology meets biophysics
}

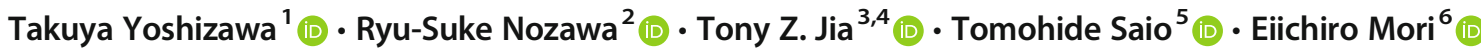

Received: 14 January 2020 / Accepted: 2 March 2020 / Published online: 18 March 2020

(C) The Author(s) 2020

\begin{abstract}
Progress in development of biophysical analytic approaches has recently crossed paths with macromolecule condensates in cells. These cell condensates, typically termed liquid-like droplets, are formed by liquid-liquid phase separation (LLPS). More and more cell biologists now recognize that many of the membrane-less organelles observed in cells are formed by LLPS caused by interactions between proteins and nucleic acids. However, the detailed biophysical processes within the cell that lead to these assemblies remain largely unexplored. In this review, we evaluate recent discoveries related to biological phase separation including stress granule formation, chromatin regulation, and processes in the origin and evolution of life. We also discuss the potential issues and technical advancements required to properly study biological phase separation.
\end{abstract}

Keywords Liquid-liquid phase separation (LLPS) · Membrane-less organelle $\cdot$ Low-complexity (LC) domain · Intrinsically disordered region/protein (IDR/IDP)

\section{The formation and regulation of membrane-less cellular organelles}

In eukaryotic evolution, cells gained a lipid bilayer nuclear membrane, separating the nucleus from the cytoplasm. Such a nuclear membrane enables segregation of certain factors (including proteins and metabolites) in their proper locations and provides selective trafficking between the nucleus and the cytoplasm. Although it may seem that the presence of a physical boundary, such as a nuclear membrane, is the only way compartments can be formed and provide trafficking control within cells, cells in fact have another option: liquid-liquid

Eiichiro Mori

emori@naramed-u.ac.jp

1 Department of Biotechnology, College of Life Sciences, Ritsumeikan University, Kusatsu, Shiga, Japan

2 Division of Experimental Pathology, Cancer Institute of the Japanese Foundation for Cancer Research (JFCR), Tokyo, Japan

3 Earth-Life Science Institute, Tokyo Institute of Technology, Tokyo, Japan

4 Blue Marble Space Institute of Science, Seattle, WA, USA

5 Department of Chemistry, Faculty of Science, Hokkaido University, Sapporo, Hokkaido, Japan

6 Department of Future Basic Medicine, Nara Medical University, Kashihara, Nara, Japan phase separation (LLPS). Indeed, a large number of organelles including nucleoli, Cajal bodies, promyelocytic leukemia (PML) bodies, processing bodies (P-bodies), and stress granules are membrane-less. It has become clear recently that these organelles are formed through LLPS (Banani et al. 2017; Shin and Brangwynne 2017). LLPS defines distinct compartments to efficiently organize cellular processes by concentrating certain factors in their proper place without interfering with one another in the complex and heterogeneous environment within a cell. These cellular bodies are dissolved during mitosis and reformed in the next round of the cell cycle (Rai et al. 2018). They are also reversible, unlike aggregates, and appear to be in a viscoelastic-dynamic fluid state, which gives them plasticity and flexibility.

Most LLPS researches are focused on events in eukaryotic cells. Even in prokaryotic cells, LLPS system may be utilized for cell division (Monterroso et al. 2016, 2019). There should be a number of undiscovered LLPS systems in both eukaryotes and prokaryotes (Alberti 2017a). As biological LLPS systems and organelles are incredibly complex and often involve a suite of co-interacting proteins, molecules, and other co-factors, we refer to the relevant interacting molecules and some of the associated processes by their commonly used abbreviations henceforth in this text. Each abbreviation is defined at first mention in the text. They are also compiled in alphabetical order by sections within this manuscript. This list, with relevant page number information, is included in Appendix Table 1 and provides readers with a quick way to 
reference the relevant abbreviations when a specific process is described in the text.

The formation of cellular membrane-less compartments is driven by multivalent interactions among nucleotides or amino acids (Banani et al. 2017; Shin and Brangwynne 2017). A disordered region of proteins termed the intrinsically disordered region (IDR), or the low-complexity (LC) domain, facilitates assembly (Kato et al. 2012; Forman-Kay and Mittag 2013; Nott et al. 2015). RNA also serves as a seed in defining the location of the phase-separated compartment. For example, the largest nuclear structure for ribosome biogenesis, the nucleolus, is formed near ribosomal RNA (rRNA) transcription sites. When rRNAs are artificially transcribed elsewhere in the chromosome, a new nucleolus-like condensate is formed at that site (Karpen et al. 1988; Oakes et al. 2006), while nucleolar component assemblies at random nuclear positions are observed in inhibiting rRNA transcription or deletion of ribosomal DNA (Berry et al. 2015; Falahati et al. 2016).

In terms of regulatory parameters for LLPS in cells, in addition to variations in the concentration of the major components that drive the LLPS, variations in microenvironments surrounding the condensates such as temperature and ionic strength are conceivable (Nott et al. 2015) as entropy and electrostatic interactions can be affected by these microenvironmental changes. Post-translational modification of proteins is also an important factor driving the assembly or dissociation of such a condensate. In particular, as the phosphate group contains negative charges, phosphorylation of proteins directly impacts the balance of multivalent electrostatic interactions (Aumiller and Keating 2016). It is interesting to note that historically, the relationship between the nucleolus and cancer has been discussed because the number and shape of nucleoli are altered in almost any type of cancer cell. An enlarged and prominent nucleolus is one of the indicators of cancer diagnoses in histopathology (Montanaro et al. 2008; Sakamoto et al. 2018). Such a phenomenon might indicate that a cancerspecific nuclear environment affects or induces the function and assembly/disassembly of condensates inside cancer cells.

\section{Stress granule assembly, regulation, and diseases}

RNA granules, composed of RNA and RNA-binding proteins (RBPs), such as stress granules (SGs), P-bodies, Cajal bodies, and nuclear speckles, play an important role in the cytoplasm and nucleus (Banani et al. 2017). LLPS of the components (proteins and RNA) drives the formation of such RNA granules, and hence, RNA granules are not surrounded by lipid membranes like other membrane bound organelles. This membrane-less feature of LLPS-driven organelles allows for quick assembly and disassembly reflecting various in-cellular conditions. RNA granules provide high-order function in complex biological systems processing or suppressing specific reactions, and unregulated RNA granules have been strongly linked to diseases. In particular, the transformation of SGs into aggregate-like inclusions is considered to be a major cause in the development of fatal neurodegenerative diseases, such as amyotrophic lateral sclerosis (ALS) and frontotemporal dementia (FTD) (Patel et al. 2015; Alberti and Dormann 2019). Proper regulation of aggregate formation is required to maintain cell homeostasis, thus suggesting that chaperones for SGs are key to the prevention of diseasecausing transformations.

In order to preserve the function of cells within an organism, these cells must survive various environmental stresses such as high temperature or oxidative stress. Under the stress conditions, SGs are formed in the cytosol in order to suppress translation. There are two reasons cells produce SGs under stress: (1) to save limited resources and energy for essential functions that more directly combat stress; and (2) to avoid increasing defective ribosomal products because of misfolding or premature termination (Patel et al. 2015; Alberti et al. 2017; Boeynaems et al. 2018). The mechanism of SG assembly occurs through self-assembly of the RBPs including G3BP (G3BP stress granule assembly factor 1 or GTPase-activating protein (SH3 domain)-binding protein 1), fused in sarcoma (FUS), and heterogeneous nuclear ribonucleoprotein A1 (hnRNPA1).

The self-assembly of FUS in relation to the LLPS mechanism has been well-studied. Kato et al. (2012) reported that cross- $\beta$ polymerization of FUS is essential for the assembly of various RNA granules. This prompted a group of researchers (Yoshizawa et al. 2018; Yoshizawa and Matsumura 2020) to apply a tag-protein system to observe FUS LLPS. In particular, they found that maltose-binding protein (MBP) prevented FUS LLPS as MBP-fused FUS was observed to be soluble and monodisperse. When a TEV protease site was introduced to MBP and FUS, droplet formations, dependent upon increasing free FUS concentration, were observed (Fig. 1a), suggesting that multivalent interactions initiated by the free $\mathrm{N}$-terminus LC domain are the mechanism resulting in the formation of RNA granules. SGs in cells are not only composed of FUS and other RBPs, but also contain mRNA, resulting in a mesh-like network between RBPs and RNAs (Ditlev et al. 2018). Thus, multivalent homo- and heterointeractions contribute to form SGs. Furthermore, SGs segregate a number of proteins including initiation factors, signaling factors, heat shock proteins, and nuclear import receptors (NIRs) (Zhang et al. 2018). These findings imply that SGs pause multiple cellular systems under stress.

Aberrant SGs have been linked to a variety of fatal diseases. In particular, uncontrolled aggregation of SG proteins is a hallmark of neurodegenerative diseases including ALS and FTD. DNA-binding protein of $43 \mathrm{kDa}$ (TDP-43), an 


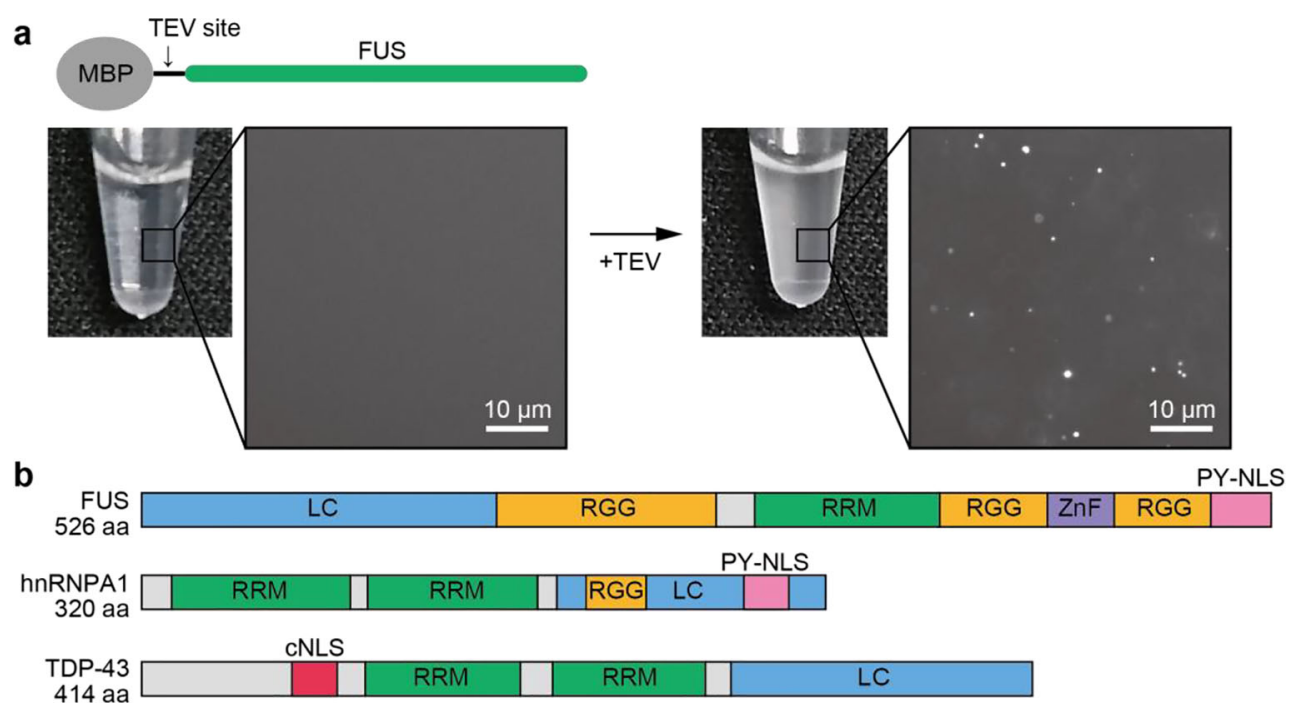

Fig. 1 a In vitro LLPS initiation of fused in sarcoma (FUS) using maltose-binding protein (MBP)-FUS. A TEV cleavage site was introduced between MBP and FUS (top). MBP-FUS is monodisperse and clear in the test tube. The solution became cloudy after TEV treatment (bottom). Close-up panels show microscope images of the solutions. b Domain map of RNA-binding proteins (RBPs) FUS, hnRNPA1, and

RBP, aggregates were observed in most ALS cases (Harrison and Shorter 2017). Mutations in other RBPs including FUS and hnRNPA1/A2 have been reported in familial neurodegenerative patients. These proteins share an LC domain adjacent to the RNA recognition motif (Fig. 1b). The LC domains are also known as prion-like domains, which natively unfold as monomers when in isolation, but polymerize upon accumulation. Although SGs utilize the self-association force with RBPs to assemble, such assemblies can form harmful amyloid-like polymers under certain conditions, especially in disease patients. Mutations found in ALS patients promote accumulation of RBP or change the physical properties (less reversibility) of SGs (Patel et al. 2015; Mateju et al. 2017). The amount of aberrant SGs increases with age and these SGs can also contain misfolded proteins.

Other than defective SG components, the hexanucleotide repeat expansion (HRE) of chromosome 9 open reading frame 72 (C9orf72) is the most common cause of familial ALS and FTD (Mori et al. 2013). In the C9orf72 HRE, the repeat sequence (GGGGCC)n is translated to dipeptide repeats by repeat-associated non-AUG (RAN) translation. Five types of dipeptides, poly glycine-proline (GP)/proline-alanine (PA)/ glycine-arginine (GR)/proline-arginine (PR)/glycine-alanine (GA), are produced from six reading frames in either the sense or the antisense strand. Poly-PR/GR peptides show strong toxicity due to their ability to target and bind to products of LLPS including nucleoli, the nuclear pore complex, and SGs, resulting in their aggregation or disassembly (Freibaum et al. 2015; Lin et al. 2016; Shi et al. 2017). Highly charged and polar poly-GR/PR peptides influence LLPS dynamics. On the other hand, poly-GP/PA has no reported toxicity and poly-GA
TDP-43 imported by nuclear import receptors (NIRs). LC, lowcomplexity domain (blue); RGG, Arginine-Glycine-Glycine repeat (orange); RRM, RNA recognition motif (green); $\mathrm{ZnF}$, zinc finger (purple); PY-NLS, proline-tyrosine nuclear localization signal (pink); cNLS, canonical-NLS (red)

has shown moderate toxicity involved in p62/ubiquitinpositive inclusions (Freibaum and Taylor 2017).

Due to the toxicity of aberrant SGs, cells require the ability to modulate the disassembly of SGs. Several studies in yeast and fruit fly models revealed that a protein quality control system is utilized to clear SG assembly. SG-recruited ATPdriven chaperone heat shock protein 70 (HSP70) can target misfolded proteins in SGs and lead to the ubiquitin proteasome or autophagy pathway, resulting in aberrant SG disassembly (Alberti et al. 2017). Recent studies have also demonstrated that NIRs play a role as a chaperone for SGs (Guo et al. 2018; Hofweber et al. 2018; Qamar et al. 2018; Yoshizawa et al. 2018). The canonical function of NIR is to transport macromolecules in the cytoplasm into the nucleus. Within the NIR group of proteins, there are twenty proteins in the human karyopherin $\beta(\operatorname{Kap} \beta)$ family with similar molecular weights (ca. 100-130 kDa) and helical topologies. Each Kap $\beta$ recognizes a specific nuclear localization signal (NLS) on macromolecules (proteins and RNA) (Soniat and Chook 2015). Importin $\alpha / \beta(\operatorname{Imp} \alpha / \beta)$ and karyopherin $\beta 2(\operatorname{Kap} \beta 2)$, subclasses of the Kap $\beta$ family of proteins, are major NIRs and many transport cargos for Imp $\alpha / \beta$ and $\operatorname{Kap} \beta 2$ have been identified. RBPs that form SGs, TDP-43 contains a canonical NLS (cNLS), which is recognized by Imp $\alpha / \beta$. FUS and hnRNPA1 contain a proline-tyrosine NLS (PY-NLS) which is recognized by Kap $\beta 2$. TDP-43, FUS, and hnRNPA1 all localize in the nucleus due to their NLS. Disease-related mutations of FUS are concentrated in the PY-NLS region, and accumulation of the nuclear protein in the cytoplasm has been observed in disease onsets. Given that Kapßs can act to dissociate selfassembly of RBPs, this suggests that the regulation performed 
by Kap $\beta$ s likely plays an important role in prevention of diseases. It also indicates the presence of additional functions of NIRs aside from their canonical functions which have yet to be explored.

In order to transport cytoplasmic cargos into the nucleus, Kap $\beta$ s pass through nuclear pore complex, a giant ring protein complex composed of multiple copies of 30 different proteins called nucleoporins. Within the ring complex is a separated phase comprising phenylalanine-glycine repeats (FG-repeats) of FG-nucleoporins. This permeability barrier typically prevents unexpected concentration-dependent diffusion of biological macromolecules between the nucleus and the cytoplasm (Schmidt and Görlich 2016). However, Kap $\beta$ s are able to penetrate nuclear pore complex by binding with the FGrepeats. One of the current authors and colleagues (Yoshizawa et al. 2018) found that NIRs utilized this function to disassemble LLPS of FUS through Kap $\beta 2$, the predominant NIR for FUS. Kap $\beta 2$ inhibits and reverses FUS LLPS binding through multiple sites including LC regions that contribute to LLPS. This chaperone function depends on the ability of Kap $\beta 2$ to bind to the PY-NLS of FUS. The location of the NLS of each RBP may also play a factor in their assembly/disassembly processes. The PY-NLS of FUS is located at the C-terminal end, while the PY-NLS of hnRNPA1 is located in the middle of the LC domain. Although the LC domain potentially contributes to LLPS through the formation of a cross- $\beta$ core for hnRNPA1, Kap $\beta 2$ can still disassemble hnRNPA1 LLPS structures, likely through direct interactions with the PYNLS, which are stronger than the LC-contributions to LLPS. However, Kap $\beta 2$ cannot disassemble TDP-43 LLPS structures since TDP-43 does not contain a PY-NLS; it instead contains a cNLS, which can be recognized only by $\operatorname{Imp} \alpha / \beta$. Unsurprisingly, Imp $\alpha / \beta$ shows chaperone function for TDP43 but not for FUS and hnRNPA1, but interestingly, Imp $\alpha / \beta$ can inhibit LLPS of chimeric FUS, where its PY-NLS has been replaced by a cNLS at the C-terminus. This suggests that as long as the NLS of a given SG-forming protein can be identified by the proper chaperone protein, chaperone activity and subsequent LLPS structure disassembly can occur. Taken together, a tight and specific interaction between NLS and NIR is required to inhibit LLPS of SG-forming RBPs. When Yoshizawa et al. (2018) performed further investigations into the relationship between FUS and Kap $\beta 2$ using NMR, they detected weak interactions between the exterior of the FUS PY-NLS and Kap $\beta 2$. This result suggests that tight binding to NLS by Kap $\beta 2$ allows Kap $\beta 2$ access to the area of the protein, which contributes to its LLPS character and results in its disassembly. Cellular model experiments also revealed that Kap $\beta 2$ expression blocks FUS accumulation in SGs. As such, the chaperone function of NIRs toward RBPs is likely important regulatory processes used by the cell to dissociate SGs in physiological conditions (Fig. 2). Nonetheless, once any of these processes become aberrant, uncontrolled aggregation of SGs may occur, leading to a variety of diseases.

Recent studies have uncovered mechanisms of regulation of SGs in both in vitro and in vivo systems, suggesting that SGs utilize the dynamic and controllable properties of LLPS to protect cells. Understanding the regulation of cellular LLPS sheds new light on our understanding of SGs. However, there is still a large gap between in vitro and in vivo studies. For example, in vivo SGs contain a number of proteins and RNAs, and the location and means by which these molecules are segregated and compartmentalized remain unclear. Posttranslational modification and other chaperones are also likely important to regulate SG states.

\section{A new view of chromatin behavior}

In higher eukaryotes, DNA is packaged into chromatin that is organized as chromosomes in the nucleus (Gilbert 2019). The organization and regulation of chromatin architecture has been studied for many years, because chromatin structure plays an important role in gene expression and genome integrity. Indeed, the alteration of chromatin structure or stability underpins many human genetic diseases and cancer.

The fundamental structural unit of chromatin is the nucleosome, which consists of negatively charged DNA containing a phosphate backbone wrapped around an octameric assembly of positively charged histone proteins (Kornberg and Lorch 1999). It is predicted that only approximately half of the negative charges of the DNA are neutralized by the positive

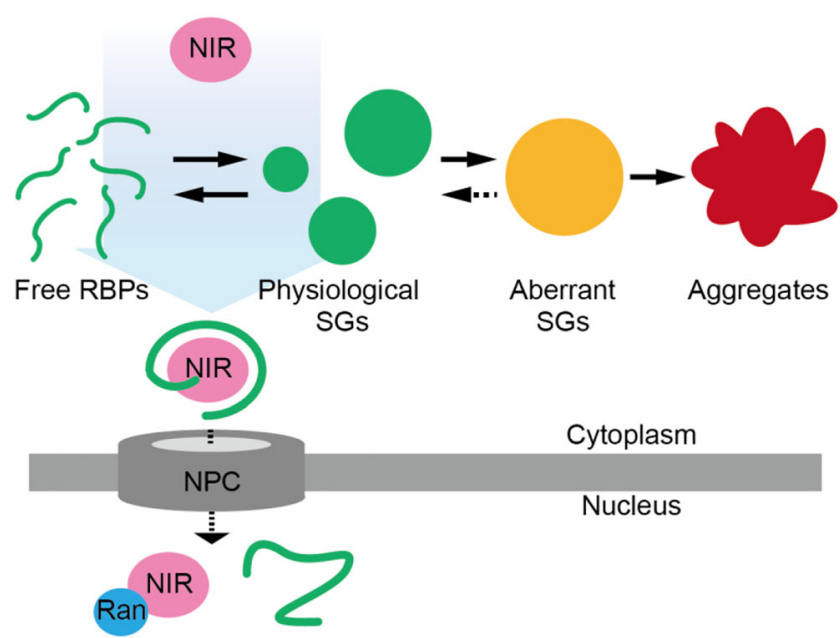

Fig. 2 Model of chaperone function of NIRs for RBPs in cells. NIRs (pink) actively carry nascently translated proteins (green lines) or proteins in physiological SGs (green circles) into the nucleus, preventing LLPS from occurring in the cytoplasm. Small GTPase Ran (blue) displaces the proteins and binds NIR in the nucleus. In the absence of this regulatory system, physiological SGs could then subsequently transform into aberrant SGs (yellow) and irreversible aggregates (red) in the cytoplasm 
charges of the histones (Khrapunov et al. 1997; Schiessel 2003; Maeshima et al. 2014). In fact, early experiments demonstrated that free cations promote self-association of chromatin (Hansen 2002); this is a crucial insight into the properties of chromatin. This process seems to leave some room for free cations and positively charged proteins, such as linker histones, to modulate local chromatin structure by binding chromatin in nucleosomes. Very recent observations indicated that reconstituted chromatin undergoes LLPS in solutions containing physiological cation concentrations (Fig. 3a, (Gibson et al. 2019)). The interaction between DNA and the histone tail enriched with positively charged amino acids drives the LLPS. In addition, LLPS is facilitated by linker histone H1, a result which is in line with early observations that histone H1 depletion causes chromatin unfolding (Allan et al. 1981) and recent biophysical approaches showing that H1-DNA forms a condensate (Turner et al. 2018). Further analysis showed that the acetylation of histone resulted in dissolution of chromatin condensates, while acetylated chromatin was triggered to rephase-separate by transcriptional regulator bromodomaincontaining protein 4 (BRD4), which recognizes the acetylated histone tail. Intriguingly, two distinct-phase, unmodified chromatin condensates and newly induced condensates adhered to each other but did not coalesce, suggesting LLPS is involved in organizing functionally distinct but physically adjacent chromatin domains in the nucleus.

The Maeshima laboratory recently provided a new view of chromatin behavior in the cell using live-cell super-resolution imaging (Nozaki et al. 2017). Their single nucleosome tracking analysis showed that nucleosomes form domains approximately $160 \mathrm{~nm}$ in diameter that behave coherently in live cells
(Fig. 3b). They suggested that the behavior of chromatin domains is like "liquid drops" rather than static and regular solidlike structures more physically constrained in nucleus. This observation led us to speculate that chromatin domains are phase-separated through self-assembly inside the nucleus and that it may provide chromatin with platform plasticity so that chromatin can conduct its many functions such as gene expression, DNA repair, and cell cycle-specific matters.

\section{Modulating chromatin structure-compaction and decompaction}

Heterochromatin is defined as a chromatin segment that is highly condensed throughout the cell cycle. It is known that abnormally rearranged heterochromatin epigenetically represses the expression of nearby genes. The molecular mechanisms for chromatin condensation and propagation of gene silencing in heterochromatin have been studied for many years (Allshire and Madhani 2018).

Heterochromatin protein 1 (HP1) was identified as a major component of heterochromatin. HP1 recognizes the trimethylation of histone $\mathrm{H} 3$ lysine 9 (H3K9me3), a hallmark of heterochromatin, through binding of the $\mathrm{N}$-terminal chromodomain (CD), and dimerizes through binding of the $\mathrm{C}$-terminal (carboxy-terminal or $\mathrm{COOH}$-terminal) chromoshadow domain (CSD) (Brasher 2000; Lachner et al. 2001). The dimerization of HP1 creates a hydrophobic surface that binds to a number of proteins containing the PxVxL (where $\mathrm{P}$ is proline, $\mathrm{V}$ is valine, $\mathrm{L}$ is leucine, and $\mathrm{x}$ is any amino-acid residue) motif (Nozawa et al. 2010). A recent
Fig. 3 A new view of chromatin behavior. a A nucleosome array forms phase-separated condensate in physiological salt concentrations facilitated by histone $\mathrm{H} 1$ (Gibson et al. 2019). b Visualizing individual nucleosomes using live-cell superresolution imaging technique suggests chromatin forms a large irregular structure behaving like "liquid drops" in the cell (Nozaki et al. 2017)
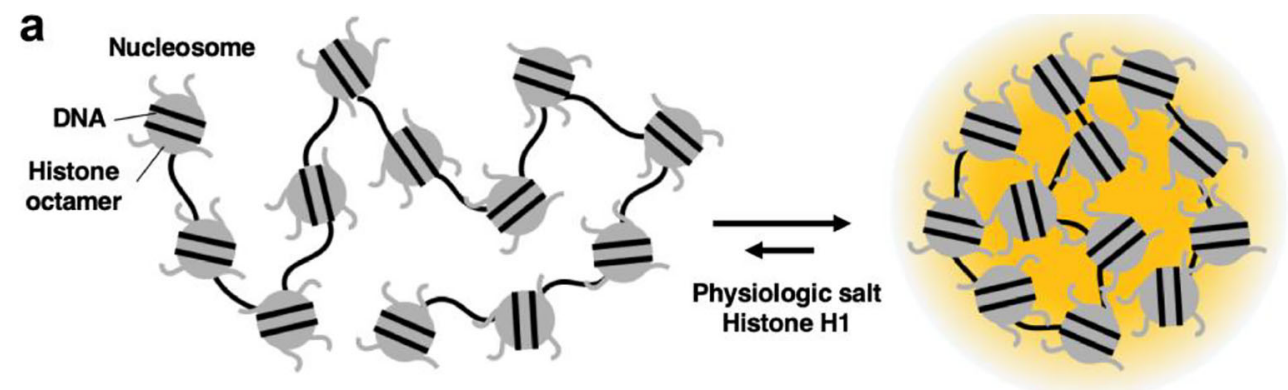

b

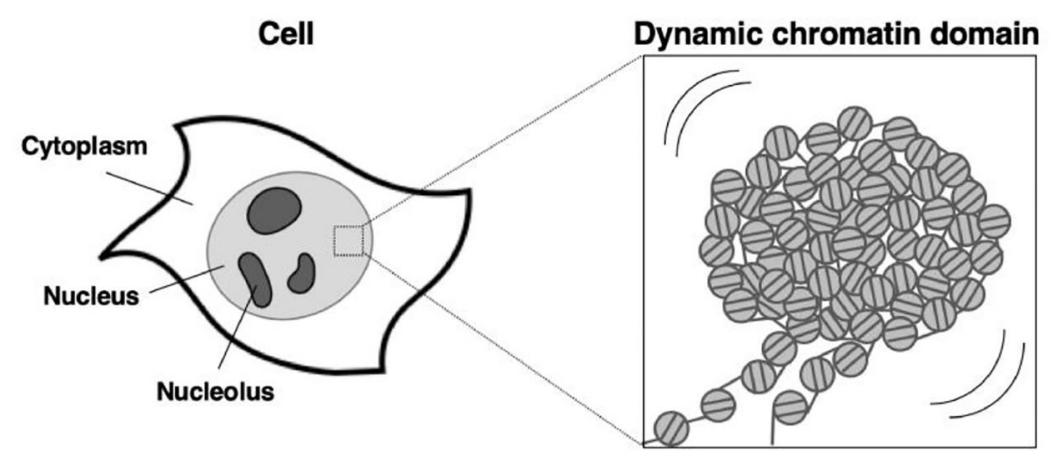


report suggested direct evidence that HP1 facilitates chromatin compaction (Sanulli et al. 2019). The CSD of the S. pombe HP1 protein, Swi6, interacts with the nucleosome core through the $\alpha$-helix of histone H2B containing a PxVxL motif. The interaction disorganizes the conformation of the histone octamer, which allows the Swi6-nucleosomal array to efficiently form phase-separated liquid condensates (Fig. 4a). This leads to a higher concentration of nucleosome within the condensates. These results suggest that Swi6 increases opportunities for multivalent interactions between nucleosomes, implying that Swi6 compacts chromatin into liquid condensates. Previous reports suggest that the mechanism of chromatin compaction would be variable among different species. The CSD of mammalian HP1 has been shown to interact with the histone $\mathrm{H} 3$ of the nucleosome core but not histone H2B. Additionally, human HP1 $\alpha$ and Drosophila HP1a themselves were reported to undergo phase separation (Larson et al. 2017; Strom et al. 2017). The LLPS structures of human HP1 $\alpha$ required the phosphorylation of the disordered N-terminal region or DNA binding, while the LLPS of Drosophila HP1a did not require such phosphorylation.

It is still unknown how large-scale chromatin structures are compacted. Recent reports give us useful insights to consider this question. A polymer-modeling simulation suggested that chromatin assembly can be induced by bridges between nucleosomes as bridging nucleosomes increases the number of chances to create additional bridges on proximal nucleosomes. This "bridging-induced attraction" or "polymer-polymer phase separation" drives a type of de-mixing of the chromatin polymer (Brackley et al. 2013; Erdel and Rippe 2018). Additionally, the CasDrop technology, i.e., inducing an LLPS on chromatin by combining clustered regularly interspaced short palindromic repeats (CRISPR) and optogenetics, demonstrated that condensates induced on targeted chromatin pull targeted loci together (Shin et al. 2018). These results lead us to imagine that HP1nucleosome condensates play a central role in the "spreading" of heterochromatin to large-scale chromatin structures. Another recent chromatin modeling study combined with Hi-C (high-throughput chromosome conformation capture) analysis suggested that interaction of heterochromatic regions, but not euchromatic regions, leads to compartmentalization of heterochromatin and euchromatin (Falk et al. 2019).

If chromatin compaction progresses in the self-organizing manner as described above, active systems to decompact chromatin would also be required for chromatin compartmentalization. Nozawa et al. have shown that scaffold attachment factor A (SAF-A) decompacts transcriptionally active largescale chromatin structures (Nozawa et al. 2017). SAF-A was originally identified as a component of the nuclear matrix and was simultaneously found as a member of the family of heterogeneous nuclear ribonucleoproteins (hnRNPs). SAF-A is a highly abundant protein and was observed to be enriched in the transcriptionally active region. Nozawa et al. found that SAF-A has an oligomerization activity that is required for chromatin decompaction. SAF-A's oligomerization is ATP dependent through SAF-A's ATPse domain, and it binds RNA through its $\mathrm{RG} / \mathrm{RGG}$ (where $\mathrm{R}$ is arginine and $\mathrm{G}$ is glycine) a

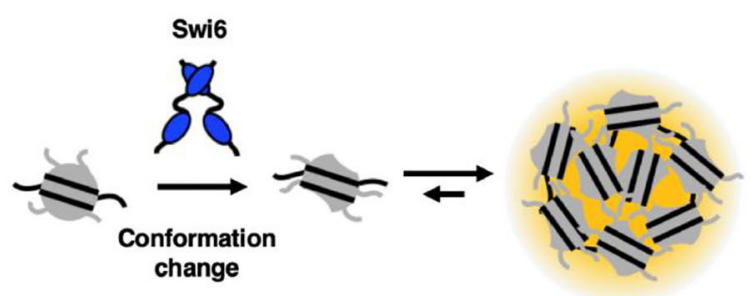

C
Splicing factor condensate

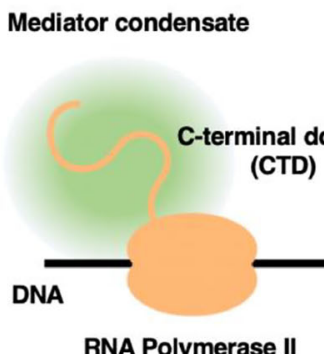

$\underset{\text { Transcription }}{\longrightarrow}$ b

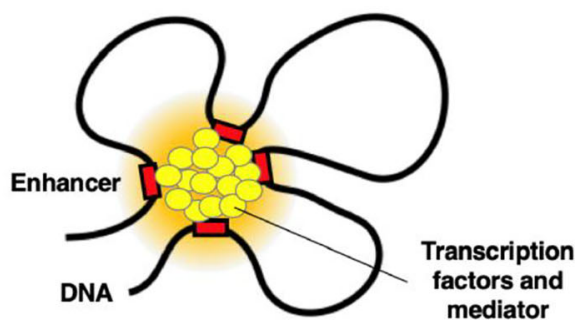
mediator
Fig. 4 The distinct microenvironment on chromatin driven by LLPS. a Schizosaccharomyces pombe HP1 protein Swi6 induces a conformation change of nucleosome, resulting in the formation of condensed, highly concentrated nucleosomes (Sanulli et al. 2019). b Super-enhancers are a cluster of enhancers bound by transcription factors and a mediator that concentrate to create a highly transcriptionally active genomic region (Hnisz et al. 2017). c Phosphorylation of the C-terminal domain (CTD) of RNA polymerase II switches its affinity from the mediator to splicing factors (Guo et al. 2019) 
motifs. It seems that SAF-A/RNA filaments biochemically form a nuclear mesh in the nucleus. Nozawa and Gilbert (2019) suggest that interactions between SAF-A and RNA form a transcriptionally responsive dynamic nuclear mesh that creates a high viscosity microenvironment keeping chromatin decompacted.

\section{Distinct microenvironment on chromatin}

The Hi-C technique, a method for quantifying pairwise chromatin interaction frequency genome-wide, revealed that the genome is organized in distinct compartments at the megabase-scale. In other words, transcriptionally active A compartments and inactive B compartments were identified (Lieberman-Aiden et al. 2009). Further studies showed that the existence of A/B compartments was consistent with replication timing (Dileep and Gilbert 2018; Takahashi et al. 2019). The molecular mechanisms responsible for compartment formation and its significance are still unclear but it seems that the organization of large-scale chromatin structure is crucial for creating distinct microenvironments to regulate chromatin functions.

It is also becoming clear that LLPS is a driving force that creates a microenvironment to facilitate chromatin functions. Super-enhancers were proposed as distinct genome regions where several enhancers, transcription factors, mediators, and RNA are locally clustered and were explained to be mediated by LLPS (Fig. 4b; Hnisz et al. 2017). In fact, RNA polymerase II (pol II), transcription factor FET (FUS/EWS [Ewing sarcoma breakpoint region 1]/TAF15 [TATA-binding protein-associated factor $2 \mathrm{~N}$ ]) family proteins, mediator complex subunit 1 (MED1), and BRD4 were shown to form condensates through LLPS and all were observed to form clusters in the nucleus (Boehning et al. 2018; Chong et al. 2018; Sabari et al. 2018). The interaction among these clusters was highly dynamic and selective (Cho et al. 2018). Another crucial observation suggested that cluster formation and interaction among clusters are regulated by phosphorylation (Kwon et al. 2013; Boehning et al. 2018; Guo et al. 2019). For example, the C-terminal domain (CTD) of pol II is a disordered low-complexity region that consists of the repetitive heptad amino acid sequence (Tyrosine, Serine, Proline, Threonine, Serine, Proline, Serine). The CTD is known to be stepwise phosphorylated in transcription processes such as initiation, elongation, and termination. The Young laboratory demonstrated that CTD phosphorylation facilitates its condensate's preference switch between MED1 and the splicing factor condensate (Fig. 4c, (Guo et al. 2019)). Altogether, these recent reports suggest that pol II CTD plays a major role as a hub to create a chromatin microenvironment by selectively associating with transcriptional factors. The association is dynamically regulated by stepwise phosphorylation of the CTD.
Contributions of LLPS to regulation of not only transcription but also DNA replication (Parker et al. 2019), DNA damage repair (Kilic et al. 2019), and mitotic progression (Trivedi et al. 2019) have also been observed, suggesting that a number of biological processes likely are conducted in spatiotemporally assembled phaseseparated microenvironments.

It should also be mentioned that RNA has various characteristics that potentially affect chromatin functions through LLPS. First, as described above, RNA serves as a seed for biomolecular assemblies. Not only does rRNA serve as a seed, but the long non-coding RNA (lncRNA) Nuclear Enriched Abundant Transcript 1 (NEAT1) acts as a seed by recruiting specific proteins, subsequently forming distinct compartments called paraspeckles (Hirose et al. 2019). NEAT1 location is simultaneously defined because the assemblies form spatially near the locus from which they are transcribed. X-inactive specific transcript (XIST) RNA, another lncRNA, has also been proposed to drive LLPS to create silent compartments for X chromosome inactivation (Cerase et al. 2019). Next, RNA buffers LLPS processes, suggesting a regulatory aspect of RNA-base LLPS systems (Maharana et al. 2018). The Hyman laboratory demonstrated that FUS, a RBP containing an LC domain, specifically forms condensates with NEAT1 while FUS condensates dissolve with higher concentrations of non-specific RNAs, suggesting that LLPS of FUS is controlled by both specific and non-specific RNAs. Moreover, the RNA itself also undergoes LLPS (Jain and Vale 2017). Expansion of short nucleotide repeats is found in several neurological and neuromuscular disorders. A transcript containing such repetitive sequences drives self-assembly through multivalent base-paring with a similar repeat number as observed in the disorders, resulting in a potential disruption of cellular functions. Finally, RNA acts as "glue" to bind various molecules and stably localize them to the same location (Ding et al. 2019). A recent compelling study in yeast demonstrated that several meiosis-specific lncRNAs and their binding proteins form condensates via LLPS on several specific loci. These condensates assist with chromosome pairing by their fusion, creating a link between homologous chromosomes at the prophase stage of meiosis. This lncRNA-based mechanism defines pairing specificity of homologous loci, preventing undesired pairing. Mouse X chromosomes were also observed to be transiently paired during $\mathrm{X}$ chromosome inactivation, a stochastic process in which a given chromosome may be inactivated with a certain probability (Masui et al. 2011). Some evidence suggests that the antisense repressor of XIST (Tsix) RNA, the antisense strand of XIST RNA, is involved in choosing whether the $\mathrm{X}$ chromosome is inactivated. This observation leads us to imagine a similar system where chromosome pairing was first developed in yeast, and then the process was conserved even upon evolution to eukaryotic cells. 


\section{LLPS processes in the origin of life}

Modern cells, ubiquitous to all life, are composed of various organelles built from assemblies of biomacromolecules. Such cells generally contain a cell membrane (usually a phospholipid bilayer also containing various cholesterols and proteins), a genetic material (DNA that can grow, replicate, and divide), and biochemical reactions which drive processes such as metabolism and replication. However, how such a cell could have emerged and evolved is still an open question. One proposal is that modern cells arose from a more primitive form of compartmentalization on early Earth (i.e., a protocell) which provided a primitive system the ability to concentrate (Keating 2012; Aumiller and Keating 2016) and segregate reactants (Jia et al. 2019). These compartments would have protected molecules from environmental degradation (ShirtEdiss et al. 2017) and provided a segregated location to allow evolution of genetic replicators without being overtaken by parasites (Bansho et al. 2016). While these could have been some of the general functions of primitive compartments, there are other more nuanced requirements for a compartment to be considered prebiotically plausible or relevant, such as the ability to allow nutrients to enter and byproducts to exit the compartment easily, as well as the ease of synthesis through prebiotically plausible mechanisms (Szostak et al. 2001). Fatty acid bilayer membrane vesicles (Mansy and Szostak 2009) are an attractive model for protocells due to their ability to encapsulate important primitive molecules such as RNA as well as their permeability to small nutrients necessary for prebiotic processes (Luisi et al. 1999; Chen and Walde 2010; Budin et al. 2014). Fatty acid membrane-based vesicle compartments which have life-like functions such as growth (Adamala and Szostak 2013a; Hentrich and Szostak 2014), replication (Adamala and Szostak 2013b), and division (Zhu and Szostak 2009; Zhu et al. 2012) have been demonstrated. While these findings appear to support such systems as the preferred prebiotic cellular framework, challenges still persist with regard to the prebiotic synthesis of some fatty acids, especially when compared with other prebiotic molecules such as amino acids, peptides, nucleosides, and, until very recently, assembly of simple vesicles composed of mixtures of fatty acids (Jordan et al. 2019). While it is clear that a lipid bilayer membrane-containing compartment was, at some point, likely the precursor of a modern cell, exactly when and how such a membrane-based primitive cell emerged is still unknown. It is possible that other forms of primitive compartmentalization preceding (or co-existing with) such membrane-bound compartments may have existed.

One potential prebiotic compartment candidate system that could have been produced on early Earth (for example, from simple peptides, nucleotides, or polyesters) exhibits "life-like" behaviors and shows compatibility with modern biomolecules having structures derived from LLPS of polymeric systems
(Mann 2012; Keating 2012; Yin et al. 2016; Matsumura et al. 2016; Jia et al. 2019). In vitro laboratory simulations of LLPS processes result in formation of co-existing liquid (or liquid-like) droplets within a bulk liquid through primarily energetically favorable processes (such as entropic effects and hydrogen bonds; (Alberti et al. 2019)) rather than through production of energy intensive covalent bonds (Pascal and Boiteau 2011). These droplet systems are membrane-less and can be composed of simple heterogeneous polymer systems more closely resembling synthetic products from early Earth, suggesting that LLPS compartments may have been able to form easily on early Earth. As discussed in other sections of this review, LLPS are ubiquitous in biology as subcellular compartments used to segregate and compartmentalize important biomolecular analytes in various biological processes (Lin et al. 2016; Alberti 2017b; Chong et al. 2018). This hints at the presence of a direct link between primitive LLPS systems and those observed in modern biology. In the following sections, we will briefly review some of the major types of prebiotically relevant LLPS systems by highlighting their composition, structure, and functions.

\section{Aqueous two-phase systems}

Aqueous two-phase systems (ATPS) are LLPS systems typically composed of one or more co-existing polymers such as poly (ethylene) glycol (PEG) and dextran. Such ATPS spontaneously separate into two distinct phases depending on the concentration of the components (the higher the concentration of both components, generally the greater the phase separation), the solution conditions, and temperature (Keating 2012; Yanagisawa et al. 2014; Iqbal et al. 2016). Upon agitation (perhaps through primitive geological processes such as wind, tides, or turbulence driven by fumaroles or other pressurized hydrothermal structures; (Chiodini et al. 2012)), the two phases rearrange into membrane-less liquid-in-liquid droplets. In the case of PEG/dextran systems, it forms dextran-rich droplets within a bulk PEG-rich phase up to tens of microns in size (Keating 2012). Over time, the droplets eventually coalesce, and the two phases separate into a droplet-less upper and lower phase. However, re-agitation will re-form the liquid-in-liquid droplet state.

While assembly of these droplets can be achieved from simple physical means, elucidation of the potential prebiotically relevant functions is still necessary before ATPS systems can be fully considered as primitive compartmentalization mechanisms. Some ATPS systems have been shown to be able to segregate biopolymers such as nucleic acids (RNA; Jia et al. 2014) and peptides (Chu and Chen 2000), resulting in increased analyte concentration (which may drive yield of primitive reactions). However, as the ATPS system does not include a membrane boundary, it 
would likely allow for free exchange of certain analytes, i.e., nutrients, between droplets depending on the droplet composition, a necessary function in early pre-biological chemical systems. Such systems can also be compartmentalized within lipid bilayer membrane vesicles, simulating a primitive celllike structure with distinct phases within the structure (Jia et al. 2014) (Fig. 5). Finally, ATPS droplets can scaffold the assembly of a layer of clay minerals, themselves an important prebiotically available geological structure promoting the synthesis and self-assembly of primitive polymers (Gillams and Jia 2018), around the droplet edge (Pir Cakmak and Keating 2017) (similar to a Pickering emulsion (Yang et al. 2017)), helping to catalyze prebiotically relevant chemical reactions and to prevent coalescence of the droplets. The addition of a clay mineral layer (and perhaps a lipid layer as well) serves to preserve each droplet's individuality (Smith and Morowitz 2016), an important property important for selective evolution of primitive replicating polymers within compartments (Bansho et al. 2016). Although PEG and dextran polymers, the most studied ATPS system in origins of life studies, may themselves not be prebiotically plausible, the ATPS droplet system still provides a model framework which can be used to study the relevant properties of ATPS systems in laboratory simulations. The fact that such membrane-less droplets can be assembled through simple physical processes by other geochemically available means on early Earth and with other prebiotically relevant systems such as oil-in-water or water-in-oil droplets suggests their possible significance as primitive compartments at the origin of life and validates their continued use as model systems to study primitive compartmentalization.

\section{Coacervate droplets}

Coacervate droplets are another membrane-less droplet structure well-represented in extant biology. Protamine (Hud et al. 1994; Balhorn et al. 2000), RNA granules (Kedersha and Anderson 2009), chromatin (Widom 1998), and nucleoli (Weber and Brangwynne 2015) are all examples of modern cellular coacervates, some of which control human disease (Shin and Brangwynne 2017). Such membrane-less coacervate droplets are fundamentally different in nature than ATPS, generally forming from interactions between two or more oppositely charged species (Priftis and Tirrell 2012) such as ATP and poly-lysine (Jia et al. 2014) or RNA and polyamines (Aumiller and Keating 2016) (Fig. 6). Initial phase separation occurs due to the immiscibility caused by a shift in the solvation energy upon binding of the analytes (Veis 2011). However, such phase separation also depends on other factors including temperature, $\mathrm{pH}$, salt concentration, and the length and concentration of the "coacervating" analytes (Priftis et al. 2013). Upon further agitation, potentially caused by primitive geological processes such as wind, tides, or turbulence driven by fumaroles or other pressurized hydrothermal structures (Chiodini et al. 2012), the separated phases
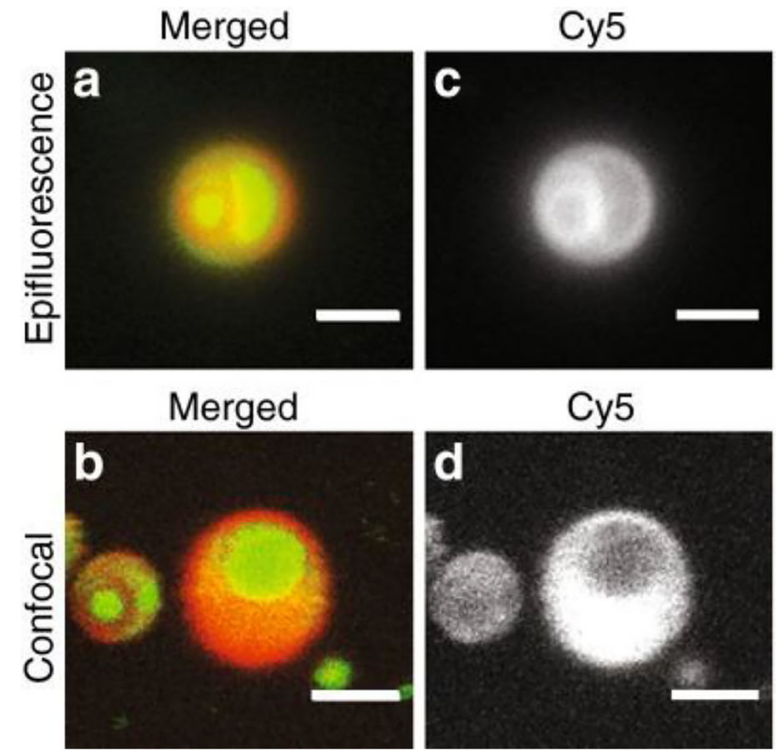

Fig. 5 Poly (ethylene) glycol (PEG)/dextran phase-separated droplets encapsulated within oleic acid vesicles. The top row shows images acquired by an epifluorescence microscope, while the bottom row shows images acquired by a confocal microscope. a, b Merged images of (c) and (e) or (d) and (f), respectively, which show separated fluorescence channels. Cyanine 5 (Cy5)-labeled RNA (red in (a) and (b)) partitioned into the outer dextran phase as shown in (c) and (d), while HTPS (8-

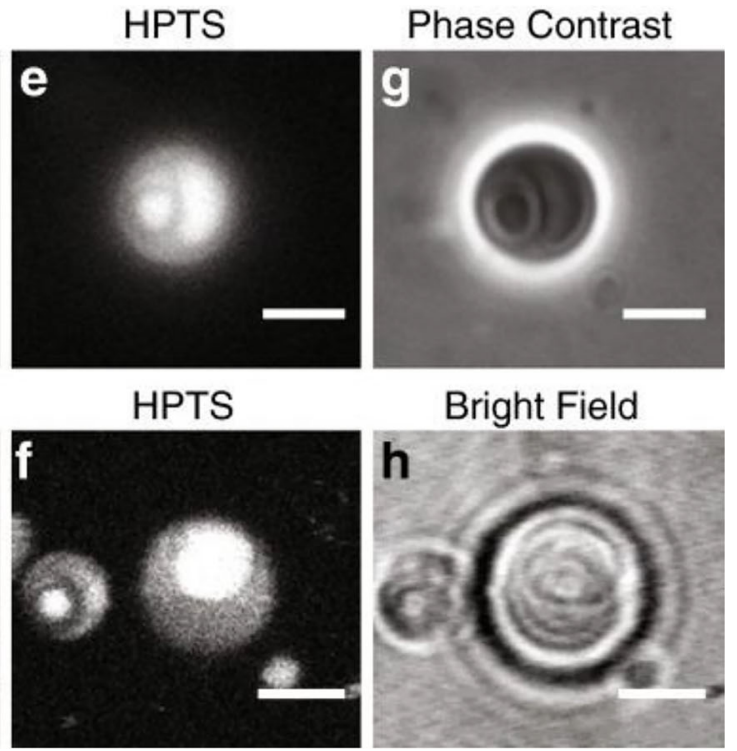

hydroxypyrene-1,3,6-trisulfonate) (green in (a) and (b)) partitioned into the PEG phase as shown in (e) and (f). $\mathbf{g}, \mathbf{h}$ Phase contrast and bright field images. Image reprinted with permission from Jia, Tony Z., Hentrich, Christian, Szostak, Jack W.: "Rapid RNA Exchange in Aqueous TwoPhase System and Coacervate Droplets." Origins of Life and Evolution of Biospheres, 44, 1-12 (2014) (Jia et al. 2014) under a Creative Commons License 
assemble into membrane-less droplets composed of a concentrated phase consisting of the bound analytes and a dilute bulk phase which is mostly aqueous.

In vitro-produced coacervates can segregate and compartmentalize important functional biomolecules (retaining their function and/or structure) such as DNA (Martin et al. 2019), RNA (Poudyal et al. 2018, 2019; Drobot et al. 2018), and proteins (Martin et al. 2016) and can scaffold the assembly of lipid layers (Tang et al. 2014). Coacervate droplets could be more permeable to small nutrients compared with fatty acid membrane vesicles and can be formed from fairly simple prebiotic components such as mononucleotides and short peptides, which potentially eliminates the need for abiotic fatty acid synthesis in primitive systems (Frankel et al. 2016). Such permeability may have been more desirable early on during the origins of life; such systems may be utilized by primitive systems to explore more possible chemistries, as opposed to more stable and selective compartments required for later biochemistries. It is also believed that the interior of a coacervate droplet more closely approximates the cellular organization and crowded environment afforded by the modern cytoplasm compared with the interior of a fatty acid vesicle (Tang et al. 2014). As such, it is possible that biological coacervate systems were ancient in origin, and a primitive coacervate could have provided primordial chemical systems a way to segregate molecules and act as a simple microreactors or even as an ancestor to modern cells themselves (Koga et al. 2011; Jia et al. 2014) while also exhibiting tunable "life-like" functions such as division or fusion (Yin et al. 2016) and heterotrophy (Qiao et al. 2017). While some of the in vitro coacervate examples presented here, mostly produced from peptide-peptide, peptide-nucleotide, peptide-nucleic acid, or polyaminenucleotide interactions, may be composed of some prebiotically implausible components, such coacervates are still a reasonable model system to study and glean information on prebiotically plausible coacervate systems, given that they share similar structures and functions. Recent research has even shown that tunable coacervates can be produced from more prebiotically plausible analytes such as 10 - to 15-residue peptides (Koga et al. 2011; Taniguchi et al. 2016; Aumiller and Keating 2016), simple polyelectrolyes like ATP (Koga et al. 2011), or simple polyamines (Aumiller and Keating 2016), perhaps leading the way for origins of life researchers to advance research in such more prebiotically plausible coacervate systems.

\section{Polyester microdroplets}

Previous research has shown that the prebiotic milieu contained a large chemical diversity including both biological (e.g., amino acids, peptides, nucleosides) and non-biological compounds of varying complexity (Guttenberg et al. 2017; Walker et al. 2017). Although much origins of life research has focused on such biological molecules, including those with fairly difficult prebiotic syntheses ( $\mathrm{Li}$ et al. 2017; Gillams and Jia 2018; Milshteyn et al. 2018; Becker et al. 2019), simple non-biological molecules abundant in such primitive chemical pools likely also directly participated in the initial emergence of these first evolving chemical systems (Forsythe et al. 2015; Chan et al. 2019). One such abundant prebiotic non-biomolecule type is alpha hydroxy acids $(\alpha \mathrm{HAs})$, a simple monomer which can be produced from prebiotically plausible mechanisms such as terrestrial spark discharge (Parker et al. 2016) or on extraterrestrial bodies such as on meteorites (Peltzer and Bada 1978). $\alpha$ HAs are very similar in structure to $\alpha$-amino acids, differing only in replacement of one amino group with a hydroxyl group, and can produce long polyester polymers via simple drying reactions (Chandru et al. 2018). Once polymerization occurs, rehydration results in assembly of a membrane-less microdroplet (Fig. 7). These microdroplets can act as primitive compartments, as shown through the segregation of various small molecule dyes, RNAs, and proteins. However, the amount
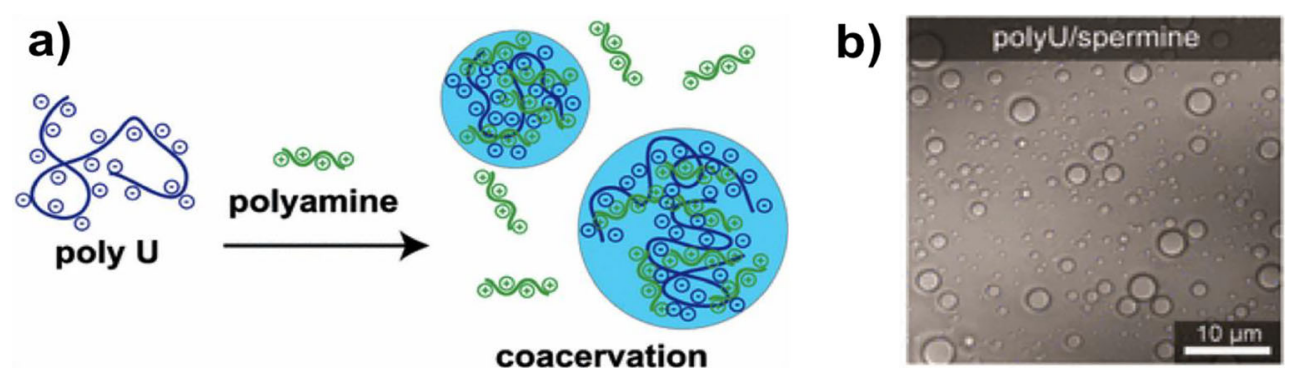

Fig. 6 Example of a coacervate droplet system composed of a nucleic acid (polyU RNA) and a simple polyamine (a). The negative charges on the RNA backbone bind strongly to the positive charges on the polyamine, resulting in a condensed coacervate phase consisting of both components (blue), and a dilute aqueous phase. b A micrograph of coacervate droplets produced from polyU and spermine. Image reprinted with permission from Aumiller, William M., Pir Cakmak,

Fatma, Davis, Bradley W., Keating, Christine D.: "RNA-Based Coacervates as a Model for Membraneless Organelles: Formation, Properties, and Interfacial Liposome Assembly" Langmuir, 32(39), 10,042-10,053 (2016), (https://doi.org/10.1021/acs.langmuir.6b02499) (Aumiller et al. 2016). Copyright American Chemical Society; further permissions related to the material excerpted should be directed to the American Chemical Society 

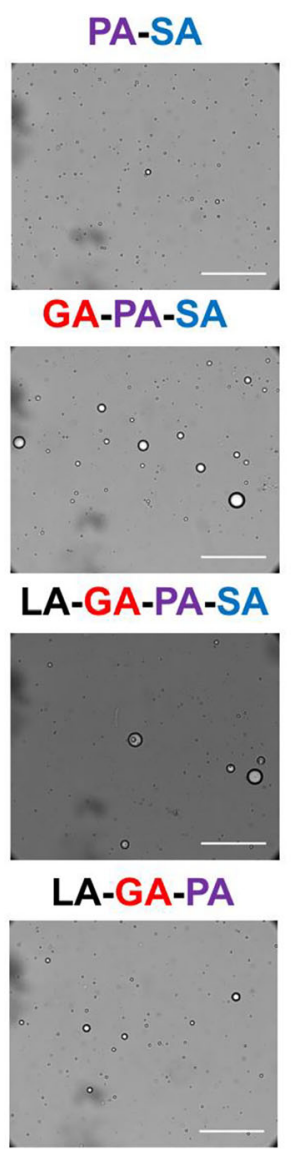

GA-PA

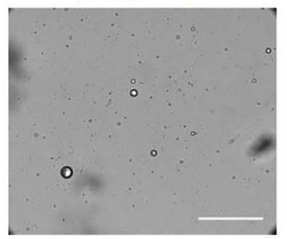

Fig. 7 Membrane-less microdroplets assembled from the rehydration of polyesters synthesized by the drying of mixtures of simple, prebiotically abundant alpha hydroxy acids. Scale bars are $100 \mu \mathrm{m}$. LA, lactic acid; GA, glycolic acid; PA, phenyllactic acid; SA, 2-hydroxy-4(methylsulfanyl) butanoic acid; MA, 2-hydroxy-4-methylpentanoic acid. The labels above each image represent the mixture present in that sample.

of free analyte exchange (stable compartmentalization) afforded varies greatly depending on the droplet chemistries and the analyte in question. In fact, protein function within and catalytic RNA (ribozyme) function in the presence of the polyester microdroplets are not inhibited and lipid layers can be scaffolded around the droplets, suggesting that these compartments are compatible with modern biologies. Mixed nonbiological/biological hybrid compartment systems incorporating LLPS structures, which themselves may have been more accessible in "messy" prebiotic environments, will be the next goal in origins of life research as they may have the potential to result in emergent properties including protection, exchange, and encapsulation of primitive components. The fact that a membrane-less compartment can be assembled from
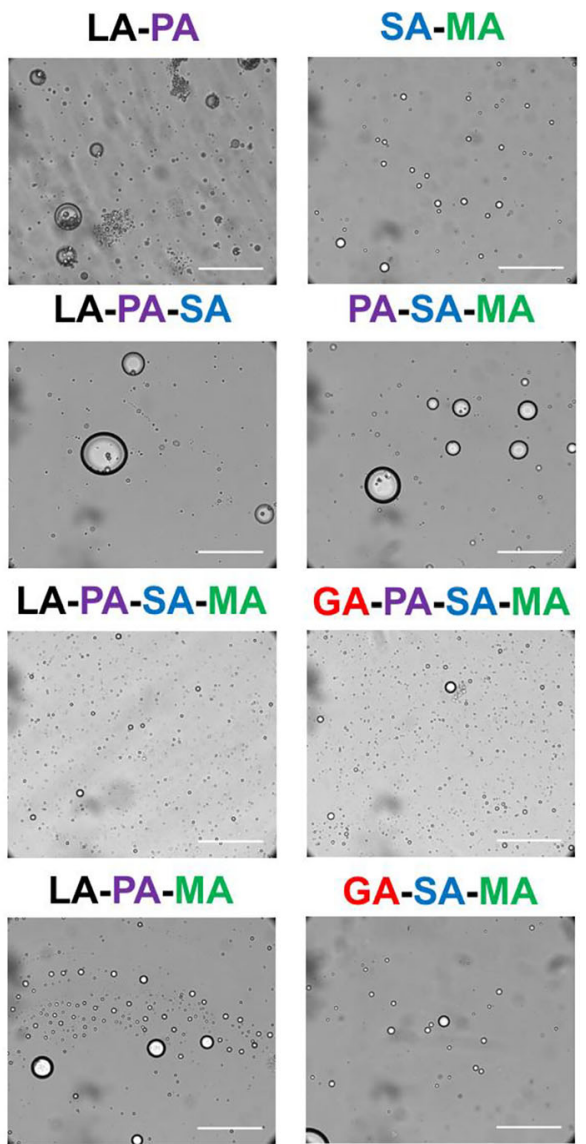

LA-GA

PA-MA
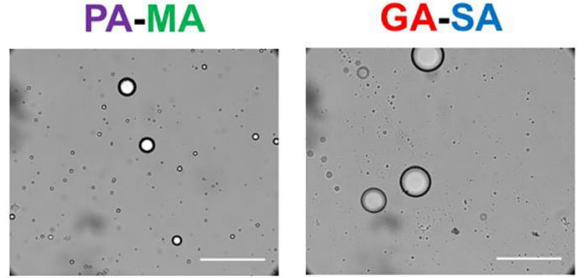

Reprinted with permission from Jia, Tony Z., Chandru, Kuhan, Hongo, Yayoi, Afrin, Rehana, Usui, Tomohiro, Myojo, Kunihiro, and Cleaves, H. James: "Membraneless polyester microdroplets as primordial compartments at the origins of life." Proceedings of the National Academy of Science of the USA, 116, 15,830-15,835 (2019) (Jia et al. 2019). Copyright Jia, Tony Z., Chandru, Kuhan, et al.

prebiotically relevant reactions of primitive molecules importantly gives evidence of the potential of a direct pathway from the "messy" prebiotic milieu into a functional primitive compartment.

\section{Techniques to observe structure and dynamics of phase-separated proteins}

Next, we summarize the techniques typically used for investigation of the structure, interactions, and dynamics of LC proteins in LLPS droplets. Although some cellular LLPS droplets and organelles contain other components, such as nucleic acids, in addition to proteins or even no proteins at 


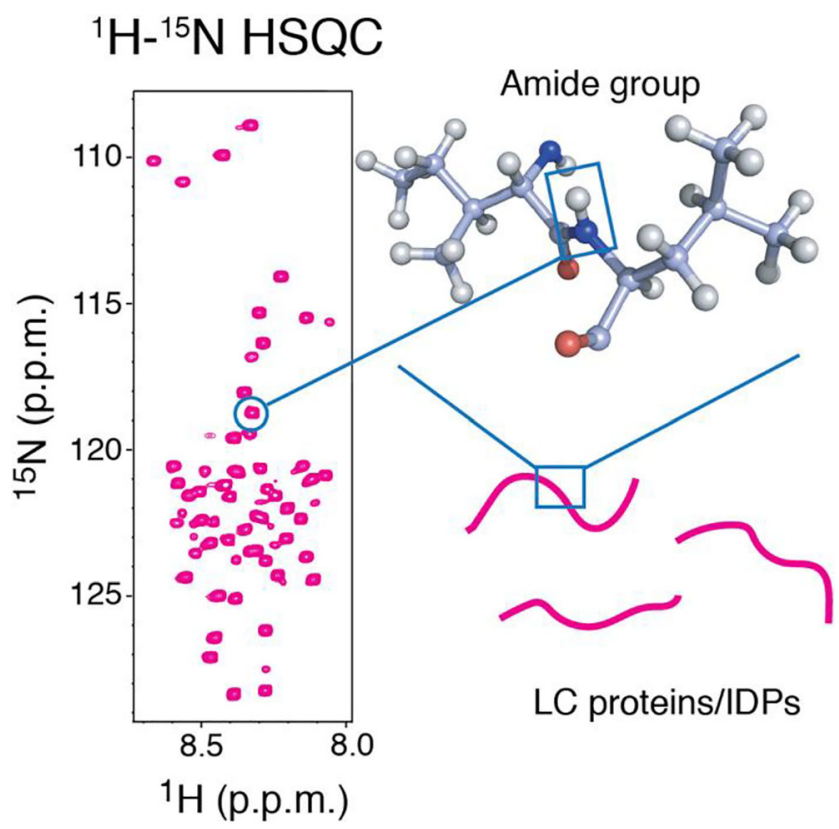

Fig. $8{ }^{1} \mathrm{H}^{15}{ }^{15}$ Heteronuclear single quantum coherence (HSQC) spectrum of a disordered protein. In principle, one amide group gives one resonance in the spectrum. If the protein exists in multiple conformational states, each of the conformational states provides distinct sets of resonances. The spectrum of LC domain proteins/IDPs typically shows a narrow chemical shift dispersion

all (in vitro ATPS and polyester systems), we focus here on the techniques that target proteins, as most cellular LLPS droplets and organelles contain at least one protein component. Light and fluorescence microscopy are frequently used to observe droplets. For example, fluorescence recovery after photobleaching (FRAP) assays evaluate the liquid property of droplets, and time-lapse imaging evaluates merging and coalescence kinetics of the droplets ( $\mathrm{Li}$ et al. 2012). Digital holographic microscopy is another potential method that can be used for 3D profiling and tracking of the LLPS droplets (Bolognesi et al. 2011; Marquet et al. 2013). Although microscopic observations of LLPS droplets provide much information about the nature of the droplet, more detailed "moleculelevel" information is required to unveil the mechanism of the formation and regulation of such droplets. Given the dynamic nature as well as the heterogeneity of proteins within LLPS droplets, probing these structures is not straightforward. Here, we highlight several key techniques used to investigate the proteins in LLPS droplets at molecular and atomic resolution.

\section{Solution nuclear magnetic resonance}

Solution nuclear magnetic resonance (NMR) is one of the most powerful tools used to investigate the structure and dynamics of protein in solution at atomic resolution. In protein solution NMR, the resonances of ${ }^{1} \mathrm{H},{ }^{15} \mathrm{~N}$, and ${ }^{13} \mathrm{C}$ are frequently observed due to their presence in protein structures, among which ${ }^{15} \mathrm{~N}$ and ${ }^{13} \mathrm{C}$ must usually be enriched by the use of isotopically labeled reagents during protein expression. The
Fig. 9 Paramagnetic effects for NMR structural studies on LC proteins. a Schematic representation of the paramagnetic effects frequently used in protein structural studies by NMR. Pseudocontact shifts (PCSs) are generated by anisotropic paramagnetic ions such as lanthanide ions, while paramagnetic relaxation enhancements (PREs) are generated by nitroxide spin labels as well as lanthanide ions. b Observation of intermolecular and intramolecular paramagnetic effects. When the paramagnetic center is attached to an isotopically labeled protein, observed paramagnetic effects represent intramolecular information (left). When the paramagnetic center is attached to an unlabeled protein, observed paramagnetic effects represent intermolecular information (right)

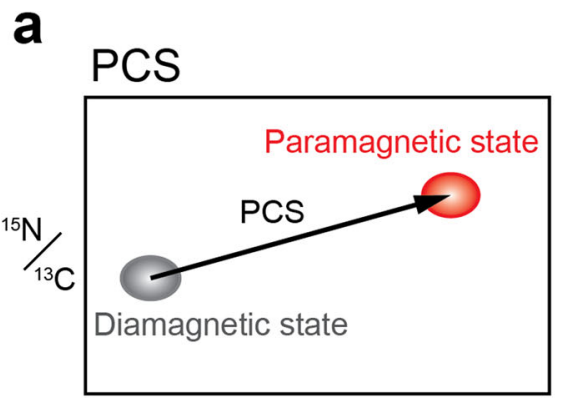

PRE

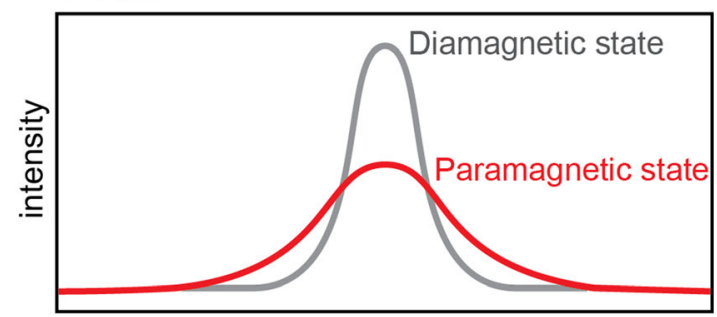

${ }^{1} \mathrm{H}$

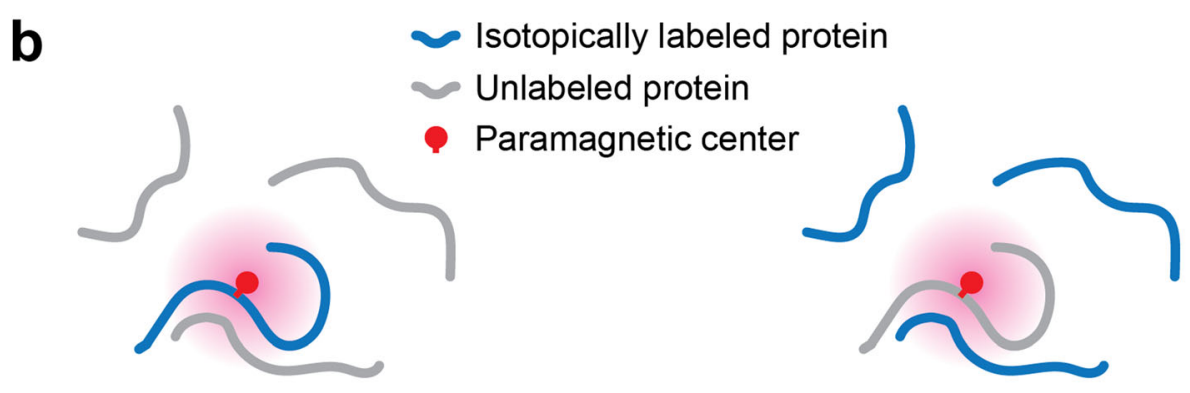

Intra-molecular effects 
use of the appropriate isotope labeling scheme enables the observation of the specific atoms in the protein. The most common experiment is $2 \mathrm{D}^{1} \mathrm{H}_{-}{ }^{15} \mathrm{~N}$ HSQC (heteronuclear single quantum coherence spectroscopy), which observes the resonances of the backbone amide group of the protein. Given that all amino acids except proline contain an amide group, the ${ }^{1} \mathrm{H}_{-}{ }^{15} \mathrm{~N}$ HSQC spectrum can be a "fingerprint" of the protein at the single-residue level (Fig. 8). A number of HSQC-based experiments investigated the interaction, structure, and dynamics of LC proteins including hnRNPA2 (Ryan et al. 2018) and FUS (Burke et al. 2015; Murthy et al. 2019), as well as other proteins in LLPS droplets (Conicella et al. 2016; Brady et al. 2017; Tsang et al. 2019). These studies reported that all such proteins have predominantly disordered conformations in LLPS droplets.

The interior of an LLPS droplet is highly viscous, and the diffusion of the FUS LC domain in the LLPS droplet was reported to be $\sim 500$ times slower than in the diluted phase (Murthy et al. 2019). Such restricted molecular motion is disadvantageous to high-resolution solution NMR. Multiple interactions between proteins can induce exchange broadening of the resonances, which further reduces the spectral sensitivity. So far, relatively sensitive NMR resonances of proteins in LLPS droplets have been reported (Burke et al. 2015; Conicella et al. 2016; Brady et al. 2017; Ryan et al. 2018; Tsang et al. 2019; Murthy et al. 2019). Typically, the dynamic nature of the LC proteins that are devoid of a stable tertiary structure is advantageous to high-sensitivity NMR observations. However, it should be noted that in NMR, smaller proteins (such as monomers) provide sharper signals, while larger species such as oligomers provide broad signals which are sometimes easily overlooked in the spectra. Especially in the case of heterogeneous conditions, such as in LLPS droplets, the possibility of the protein size-dependence of the NMR resonances must be considered. The use of NMR experiments that monitor the mobility of the protein, such as pulsed-field gradient (PFG) NMR, can assist in LLPS droplet protein analyses (Murthy et al. 2019); PFG NMR allows the separation of the resonances according to the difference in the translational diffusion coefficients (Pagès et al. 2017). In principle, PFG NMR is able to distinguish the different oligomeric species, but still have difficulty detecting the larger species due to resonance broadening.

In order to increase the spectral sensitivity for large oligomers and/or proteins in a viscous LLPS droplet, techniques utilizing isotope labeling are beneficial. In NMR of slowtumbling molecules, the transverse relaxation time of the observed nuclei becomes too short to obtain high-resolution NMR spectra, which is one of the current limits of NMR analysis of LLPS proteins. Labeling techniques exploiting ${ }^{2} \mathrm{H}$ reduce the transverse relaxation time of the observed nuclei, and thus extend the target protein size limit for NMR observation. Exploitation of methyl-selective isotope labeling combined with methyl-transverse relaxation optimized spectroscopy (TROSY) enabled researchers to obtain high-resolution NMR spectra of the 1 MDa proteasome (Mainz et al. 2013). In methyl-selective labeling, the methyl groups of Ile, Leu, Val, Met, Ala, and Thr are specifically labeled as ${ }^{13} \mathrm{C}^{1} \mathrm{H}_{3}$; other hydrogens in the protein are deuterated, resulting in prolonged transverse relaxation times which provide sharper resonance peaks even for large proteins (Saio et al. 2014). Although the rotational correlation time of the $1 \mathrm{MDa}$ proteasome (rotational correlation time, $\tau_{c} 590 \mathrm{~ns}$ ) is much longer than that of small proteins (e.g., $\tau_{c} \sim 15 \mathrm{~ns}$ for a $\sim 25 \mathrm{kDa}$ grobular protein) and cannot be observed in typical NMR conditions, methyl-based NMR techniques effectively enable high-resolution NMR analysis. These NMR techniques will be beneficial in the future in the analysis of polymers of the LC proteins and LLPS chaperones such as Kapß2 (100 kDa) (Yoshizawa et al. 2018).

Paramagnetic probes are of great use in NMR structural analysis of LC proteins in LLPS droplets. A paramagnetic center, such as a nitroxide spin label, and paramagnetic metal ions including trivalent lanthanide ions can be introduced to the protein of interest via a disulfide bond through a cysteine residue (Saio and Ishimori 2019). The benefit of paramagnetic probes is their ability to assist in observation of long-range structural information of the observed nuclei in the protein. The paramagnetic center induces a variety of paramagnetic effects including paramagnetic relaxation enhancement (PRE) and pseudocontact shift (PCS). PREs provide distance information of nuclei within the range of $<25 \sim 30 \AA$ from the paramagnetic center, and PCSs provide distance and angular information of nuclei within the range of $<\sim 40 \AA$ (Fig. 9). Intramolecular and intermolecular long-range structural information is beneficial to infer the conformation and oligometric state of LC proteins. For example, when a paramagnetic center is introduced to an isotopically labeled protein mixed with an excess of unlabeled protein, the observed paramagnetic effects provide intramolecular information (i.e., the conformational state of the protein). When a paramagnetic center is introduced to an unlabeled protein mixed with an excess of isotopically labeled protein, the observed paramagnetic effects provide intermolecular information (i.e., the oligomeric state and interaction) (Fig. 9). While the use of PRE has been used in NMR-based structural analyses of LC proteins (Conicella et al. 2016; Ryan et al. 2018; Murthy et al. 2019), other paramagnetic effects have not yet been utilized. Exploitation of the other available paramagnetic effects including PCS and residual dipolar coupling (RDC) will further extend the possibilities of and increase the gleanable information from NMR studies of proteins in LLPS droplets.

\section{Electron paramagnetic resonance}

In addition to NMR, electron paramagnetic resonance (EPR) has been used to monitor the dynamics of proteins in LLPS 
droplets (Babinchak et al. 2019). The mobility of the sitedirected spin label (SDSL) attached to specific positions of the LC protein is evaluated on basis of the EPR spectra. Since EPR signals are highly sensitive to local motions of the paramagnetic spin label attached to the protein, the EPR spectra provide information about protein dynamics. Based on this, the oligomerization of the transactive response TDP-43 in LLPS droplets was investigated (Babinchak et al. 2019). Further developments in EPR techniques of late include distance measurement by pulsed EPR, double electron-electron resonance (DEER) in which the distance and population between paramagnetic centers can be evaluated (Jeschke 2012). With DEER measurements, the structure of the oligomeric species of LC proteins in LLPS droplets can be further investigated. Given this recent development and additional expected developments of EPR techniques, further application of EPR toward LLPS analysis is expected and needed.

\section{Other biochemical and biophysical methods}

In addition to spectroscopic tools, other methods to study proteins within LLPS droplets have been applied. A biochemical method exploiting chemical modification and mass spectroscopy, termed chemical footprinting, which allows identification of the structure of LC proteins in LLPS droplets was conducted (Xiang et al. 2015). Chemical footprinting utilizes $\mathrm{N}$-acetylimidazole (NAI) as a reactive chemical compound that acetylates the side chains of Ser, Tyr, Lys, Thr, Arg, and Asn. By evaluating the residue-by-residue NAI reactivity by stable isotope labeling by/with amino acids in cell culture (SILAC) mass spectrometry, the exposure of each amino acid residue within an LLPS structure to solution can be estimated. Using this method, the existence of the cross- $\beta$ polymer of the hnRNPA2 LC domain in LLPS droplets was discovered (Xiang et al. 2015).

High-speed atomic force microscopy (AFM) is another promising future tool that can be used to investigate LC proteins. AFM surveys the structure of a protein weakly immobilized onto a sample stage substrate by tapping the protein using a cantilever (Ando et al. 2013). High-speed AFM provides real-time video of the protein in solution at nm-resolution on the second or sub-second timescale, and has been used to investigate the polymers of LC proteins (Babinchak et al. 2019) and gels of protein fibers (Cui et al. 2019; Wang et al. 2019). More recently, high-speed AFM has been utilized in the observation of the protein in the LLPS droplet (Fujioka et al. 2020).

For structure determination of polymers of LC proteins, exploitation of solid-state NMR (Murray et al. 2017; Murray and Tycko 2020), X-ray diffraction, and micro-electron diffraction (micro-ED) (Guenther et al. 2018; Luo et al. 2018) have been applied. Formation of the cross- $\beta$ polymer was also observed for the FUS LC domain in the solid phase through solid-state NMR (Murray et al. 2017; Murray and Tycko 2020). In addition to single particle cryo-electron microscopy (cryo-EM) analysis, micro-ED is one of the recent emerging techniques in the field of structural biology (Nannenga and Gonen 2019). In micro-ED, the electron diffraction is collected for micro-3D protein crystals. Since only a-fewmicrometer-size crystals give high-resolution electron density maps, micro-ED reduces the requirement crystal size for structure determination of proteins. We believe the next step is to try applying these electron-based imaging techniques in studies on proteins in LLPS droplets.

\section{Conclusion}

In recent decades, there has been increased knowledge on the crucial role biological phase separation plays in bridging several gaps. It has addressed the gap between molecular biology at the genetic level, cell biology at the cellular level, and structural biology at the atomic level. While more and more biological processes can now be explained by LLPS phenomena, a large knowledge gap still remains with regard to understanding cellular LLPS. Further development of detection methods and novel approaches is required to better understand biological process effected and affected through biological phase separation.

Acknowledgments The authors thank Keren-Happuch E for the critical reading of the manuscript.

Funding information T.Z.J. is a researcher at the Earth-Life Science Institute at Tokyo Institute of Technology, which is a member of the World Premier International Research Center Initiative (WPI) and is supported in part by the Japanese Ministry of Education, Culture, Sports, Science and Technology (MEXT). R.-S. N. is supported by the Leading Initiative for Excellent Young Researchers, MEXT, Japan. This work was supported by grants from AMED Brain/MINDS Beyond [JP19dm0307032] to E.M., JSPS KAKENHI [JP18K14354 to T.Z.J., JP17H07031 to E.M., JP19H04945, JP19K06504, JP18H05229, JP17H05657, JP17H05867 to T.S., JP19K16060 to T.Y], Japan Astrobiology Center Project Grant AB311021 to T.Z.J., Takeda Science Foundation to E.M. and T.S., Kanzawa Medical Research Foundation to E.M., Uehara Memorial Foundation to E.M., Nakatomi Foundation to E.M., Konica Minolta Science and Technology Foundation to E.M., Naito Foundation to E.M., MSD Life Science Foundation to E.M., Mochida Memorial Foundation for Medical and Pharmaceutical Research to E.M., SENSHIN Medical Research Foundation to E.M., Terumo Foundation for Life Sciences and Arts to E.M., Nara Kidney Disease Research Foundation to E.M., Novartis Research Grants to E.M., Akiyama Life Science Foundation Grants-in-Aid to T.S., Northern Advancement Center for Science and Technology Grants-inAid to T.S., Astellas Foundation for Research on Metabolic Disorders to T.S., Sumitomo Foundation to T.S., Senri Life Science Foundation to T.S., Daiichi Sankyo Foundation of Life Science to T.Y., Nakabayashi Trust For ALS Research to T.Y., and by unrestricted funds provided to E.M. from Dr. Taichi Noda (KTX Corp., Aichi, Japan) and Dr. Yasuhiro Horii (Koseikai, Nara, Japan). 


\section{Compliance with ethical standards}

Conflict of interest The authors declare that they have no conflict of interest.

\section{Appendix}

Table 1 List of relevant abbreviations by section

\begin{tabular}{|c|c|}
\hline $\begin{array}{l}\text { The formation and regulation of membrane-less cellular } \\
\text { organelles }\end{array}$ & pp. 2 \\
\hline Abbreviation & Full description \\
\hline IDR & Intrinsically disordered region \\
\hline LC domain & Low-complexity domain \\
\hline LLPS & Liquid-liquid phase separation \\
\hline P-body & Processing body \\
\hline PML Body & Promyoelocytic leukemia body \\
\hline rRNA & Ribosomal RNA \\
\hline Stress granule assembly, regulation, and diseases & pp. 2-4 \\
\hline Abbreviation & Full description \\
\hline ALS & Amyotrophic lateral sclerosis \\
\hline C9orf72 & Chromosome 9 open reading frame 72 \\
\hline cNLS & Canonical NLS \\
\hline FG-repeats & Phenylalanine-Glycine repeats \\
\hline FUS & Fused in sarcoma \\
\hline G3BP1 & $\begin{array}{l}\text { G3BP stress granule assembly factor } 1 \text { or GTPase-activating protein (SH3 domain)-- } \\
\text { binding protein } 1\end{array}$ \\
\hline GA & Glycine-Alanine \\
\hline GP & Glycine-Proline \\
\hline GR & Glycine-Arginine \\
\hline hnRNPA1 & Heterogeneous nuclear ribonucleoprotein A1 \\
\hline HRE & Hexanucleotide repeat expansion \\
\hline HSP70 & Heat shock protein 70 \\
\hline $\operatorname{Imp} \alpha / \beta$ & Importin $\alpha / \beta$ \\
\hline Kap $\beta$ & Karyopherin $\beta$ \\
\hline Кар $\beta 2$ & Karyopherin $\beta 2$ \\
\hline MBP & Maltose-binding protein \\
\hline NIR & Nuclear import receptor \\
\hline NLS & Nuclear localization signal \\
\hline $\mathrm{PA}$ & Proline-Alanine \\
\hline PR & Proline-Arginine \\
\hline PY-NLS & Proline-tyrosine NLS \\
\hline RBP & RNA-binding protein \\
\hline SG & Stress granule \\
\hline TDP-43 & Transactive response DNA-binding protein of $43 \mathrm{kDa}$ \\
\hline A new view of chromatin behavior & pp. 4-5 \\
\hline Abbreviation & Full description \\
\hline BRD4 & Bromodomain-containing protein 4 \\
\hline $\begin{array}{l}\text { Modulating chromatin structure-compaction and } \\
\text { decompaction }\end{array}$ & pp. 5 \\
\hline Abbreviation & Full description \\
\hline C-terminal & Carboxy-terminal or $\mathrm{COOH}$-terminal \\
\hline
\end{tabular}


Table 1 (continued)

\begin{tabular}{|c|c|}
\hline $\mathrm{CD}$ & Chromodomain \\
\hline CRISPR & Clustered regularly interspaced short palindromic repeats \\
\hline CSD & Chromoshadow domain \\
\hline H3K9me3 & Trimethylation of histone $\mathrm{H} 3$ lysine 9 \\
\hline $\mathrm{Hi}-\mathrm{C}$ & High-throughput chromosome conformation capture \\
\hline hnRNPs & Heterogeneous nuclear ribonucleoproteins \\
\hline HP1 & Heterochromatin protein 1 \\
\hline SAF-A & Scaffold attachment factor A \\
\hline Swi6 & Chromoshadow domain of the $S$. pombe HP1 protein \\
\hline Distinct microenvironment on chromatin & pp. 6 \\
\hline Abbreviation & Full description \\
\hline CTD & C-terminal domain \\
\hline EWS & Ewing sarcoma breakpoint region 1 \\
\hline FET family proteins & FUS, EWS, and TAF15 \\
\hline $\operatorname{lncRNA}$ & Long non-coding RNA \\
\hline MED1 & Mediator complex subunit 1 \\
\hline NEAT1 & Nuclear Enriched Abundant Transcript 1 \\
\hline pol II & RNA polymerase II \\
\hline TAF15 & TATA-binding protein-associated factor $2 \mathrm{~N}$ \\
\hline Tsix & The antisense repressor of XIST \\
\hline XIST RNA & $\mathrm{X}$-inactive specific transcript RNA \\
\hline Aqueous two-phase systems & pp. $7-8$ \\
\hline Abbreviation & Full description \\
\hline ATPS & Aqueous two-phase system \\
\hline PEG & poly (ethylene) glycol \\
\hline Polyester microdroplets & pp. 8-9 \\
\hline Abbreviation & Full description \\
\hline$\alpha \mathrm{HA}$ & Alpha hydroxy acid \\
\hline $\begin{array}{l}\text { Techniques to observe structure and dynamics of phase- } \\
\text { separated proteins }\end{array}$ & pp. $8-9$ \\
\hline Abbreviation & Full description \\
\hline FRAP & Fluorescence recovery after photobleaching \\
\hline Solution nuclear magnetic resonance (NMR) & pp. $9-10$ \\
\hline Abbreviation & Full description \\
\hline HSQC & Heteronuclear single quantum coherence spectroscopy \\
\hline NMR & Nuclear magnetic resonance \\
\hline PCS & Pseudocontact shift \\
\hline PFG-NMR & Pulsed-field gradient-nuclear magnetic resonance \\
\hline PRE & Paramagnetic relaxation enhancement \\
\hline $\mathrm{RDC}$ & Residual dipolar coupling \\
\hline TROSY & Transverse relaxation optimized spectroscopy \\
\hline Electron paramagnetic resonance (EPR) & pp. 10 \\
\hline Abbreviation & Full description \\
\hline DEER & Double electron-electron resonance \\
\hline EPR & Electron paramagnetic resonance \\
\hline SDSL & Site-directed spin label \\
\hline Other biochemical and biophysical methods & pp. $10-11$ \\
\hline Abbreviation & Full description \\
\hline AFM & Atomic force microscopy \\
\hline Cryo-EM & Cryo-electron microscopy \\
\hline Micro-ED & Micro-electron diffraction \\
\hline NAI & N-acetylimidazole \\
\hline SILAC & Stable isotope labeling by/with amino acids in cell culture \\
\hline
\end{tabular}


Open Access This article is licensed under a Creative Commons Attribution 4.0 International License, which permits use, sharing, adaptation, distribution and reproduction in any medium or format, as long as you give appropriate credit to the original author(s) and the source, provide a link to the Creative Commons licence, and indicate if changes were made. The images or other third party material in this article are included in the article's Creative Commons licence, unless indicated otherwise in a credit line to the material. If material is not included in the article's Creative Commons licence and your intended use is not permitted by statutory regulation or exceeds the permitted use, you will need to obtain permission directly from the copyright holder. To view a copy of this licence, visit http://creativecommons.org/licenses/by/4.0/.

\section{References}

Adamala K, Szostak JW (2013a) Competition between model protocells driven by an encapsulated catalyst. Nat Chem 5:495-501. https:// doi.org/10.1038/nchem.1650

Adamala K, Szostak JW (2013b) Nonenzymatic template-directed RNA synthesis inside model protocells. Science 342(80):1098-1100. https://doi.org/10.1126/science.1241888

Alberti S (2017a) The wisdom of crowds: regulating cell function through condensed states of living matter. J Cell Sci 130:2789-2796. https:// doi.org/10.1242/jcs.200295

Alberti S (2017b) Phase separation in biology. Curr Biol 27:R1097R1102. https://doi.org/10.1016/j.cub.2017.08.069

Alberti S, Dormann D (2019) Liquid-liquid phase separation in disease. Annu Rev Genet 53:1-24. https://doi.org/10.1146/annurev-genet112618-043527

Alberti S, Mateju D, Mediani L, Carra S (2017) Granulostasis: protein quality control of RNP granules. Front Mol Neurosci 10:1-14. https://doi.org/10.3389/fnmol.2017.00084

Alberti S, Gladfelter A, Mittag T (2019) Considerations and challenges in studying liquid-liquid phase separation and biomolecular condensates. Cell 176:419-434. https://doi.org/10.1016/j.cell.2018.12.035

Allan J, Cowling GJ, Harborne N et al (1981) Regulation of the higherorder structure of chromatin by histones $\mathrm{H} 1$ and H5. J Cell Biol 90: 279-288. https://doi.org/10.1083/jcb.90.2.279

Allshire RC, Madhani HD (2018) Ten principles of heterochromatin formation and function. Nat Rev Mol Cell Biol 19:229-244. https://doi. org/10.1038/nrm.2017.119

Ando T, Uchihashi T, Kodera N (2013) High-speed AFM and applications to biomolecular systems. Annu Rev Biophys 42:393-414. https://doi.org/10.1146/annurev-biophys-083012-130324

Aumiller WM, Keating CD (2016) Phosphorylation-mediated RNA/ peptide complex coacervation as a model for intracellular liquid organelles. Nat Chem 8:129-137. https://doi.org/10.1038/nchem. 2414

Aumiller WM, Pir Cakmak F, Davis BW, Keating CD (2016) RNA-based coacervates as a model for membraneless organelles: formation, properties, and interfacial liposome assembly. Langmuir 32: 10042-10053. https://doi.org/10.1021/acs.langmuir.6b02499

Babinchak WM, Haider R, Dumm BK et al (2019) The role of liquidliquid phase separation in aggregation of the TDP-43 low-complexity domain. J Biol Chem 294:6306-6317. https://doi.org/10.1074/ jbc.RA118.007222

Balhorn R, Brewer L, Corzett M (2000) DNA condensation by protamine and arginine-rich peptides: analysis of toroid stability using single DNA molecules. Mol Reprod Dev 56:230-234. https://doi.org/10. 1002/(SICI)1098-2795(200006)56:2+<230::AID-MRD3>3.0.CO; $2-\mathrm{V}$
Banani SF, Lee HO, Hyman AA, Rosen MK (2017) Biomolecular condensates: organizers of cellular biochemistry. Nat Rev Mol Cell Biol 18:285-298. https://doi.org/10.1038/nrm.2017.7

Bansho Y, Furubayashi T, Ichihashi N, Yomo T (2016) Host-parasite oscillation dynamics and evolution in a compartmentalized RNA replication system. Proc Natl Acad Sci 113:4045-4050. https://doi. org/10.1073/pnas.1524404113

Becker S, Feldmann J, Wiedemann S et al (2019) Unified prebiotically plausible synthesis of pyrimidine and purine RNA ribonucleotides. Science 366(80):76-82. https://doi.org/10.1126/science.aax2747

Berry J, Weber SC, Vaidya N et al (2015) RNA transcription modulates phase transition-driven nuclear body assembly. Proc Natl Acad Sci U S A 112:E5237-E5245. https://doi.org/10.1073/pnas. 1509317112

Boehning M, Dugast-Darzacq C, Rankovic M et al (2018) RNA polymerase II clustering through carboxy-terminal domain phase separation. Nat Struct Mol Biol 25:833-840. https://oi.org/10.1038/ s41594-018-0112-y

Boeynaems S, Alberti S, Fawzi NL et al (2018) Protein phase separation: a new phase in cell biology. Trends Cell Biol 28:420-435. https:// doi.org/10.1016/j.tcb.2018.02.004

Bolognesi G, Bianchi S, Di Leonardo R (2011) Digital holographic tracking of microprobes for multipoint viscosity measurements. Opt Express 19:19245. https://doi.org/10.1364/OE.19.019245

Brackley CA, Taylor S, Papantonis A et al (2013) Nonspecific bridginginduced attraction drives clustering of DNA-binding proteins and genome organization. Proc Natl Acad Sci U S A 110. https://doi. org/10.1073/pnas.1302950110

Brady JP, Farber PJ, Sekhar A et al (2017) Structural and hydrodynamic properties of an intrinsically disordered region of a germ cellspecific protein on phase separation. Proc Natl Acad Sci 114: E8194-E8203. https://doi.org/10.1073/pnas.1706197114

Brasher SV (2000) The structure of mouse HP1 suggests a unique mode of single peptide recognition by the shadow chromo domain dimer. EMBO J 19:1587-1597. https://doi.org/10.1093/emboj/19.7.1587

Budin I, Prywes N, Zhang N, Szostak JW (2014) Chain-length heterogeneity allows for the assembly of fatty acid vesicles in dilute solutions. Biophys J 107:1582-1590. https://doi.org/10.1016/j.bpj.2014. 07.067

Burke KA, Janke AM, Rhine CL, Fawzi NL (2015) Residue-by-residue view of in vitro FUS granules that bind the C-terminal domain of RNA polymerase II. Mol Cell 60:231-241. https://doi.org/10.1016/ j.molcel.2015.09.006

Cerase A, Armaos A, Neumayer C et al (2019) Phase separation drives Xchromosome inactivation: a hypothesis. Nat Struct Mol Biol 26: 331-334. https://doi.org/10.1038/s41594-019-0223-0

Chan MA, Hinman NW, Potter-McIntyre SL et al (2019) Deciphering biosignatures in planetary contexts. Astrobiology 19:1075-1102. https://doi.org/10.1089/ast.2018.1903

Chandru K, Guttenberg N, Giri C et al (2018) Simple prebiotic synthesis of high diversity dynamic combinatorial polyester libraries. Commun Chem 1:30. https://doi.org/10.1038/s42004-018-0031-1

Chen IA, Walde P (2010) From self-assembled vesicles to protocells. Cold Spring Harb Perspect Biol 2:1-14. https://doi.org/10.1101/ cshperspect.a002170

Chiodini G, Caliro S, Lowenstern JB et al (2012) Insights from fumarole gas geochemistry on the origin of hydrothermal fluids on the Yellowstone Plateau. Geochim Cosmochim Acta 89:265-278. https://doi.org/10.1016/j.gca.2012.04.051

Cho W-K, Spille J-H, Hecht M et al (2018) Mediator and RNA polymerase II clusters associate in transcription-dependent condensates. Science 361(80):412-415. https://doi.org/10.1126/science.aar4199

Chong S, Dugast-Darzacq C, Liu Z et al (2018) Imaging dynamic and selective low-complexity domain interactions that control gene transcription. Science 361(80):eaar2555. https://doi.org/10.1126/ science.aar 2555 
Chu IM, Chen WY (2000) Partition of amino acids and peptides in aqueous two-phase systems. Methods Biotechnol 11:95-105. https://doi. org/10.1016/B978-0-08-088504-9.00124-0

Conicella AE, Zerze GH, Mittal J, Fawzi NL (2016) ALS mutations disrupt phase separation mediated by $\alpha$-helical structure in the TDP-43 low-complexity C-terminal domain. Structure 24:15371549. https://doi.org/10.1016/j.str.2016.07.007

Cui M, Wang X, An B et al (2019) Exploiting mammalian lowcomplexity domains for liquid-liquid phase separation-driven underwater adhesive coatings. Sci Adv 5:1-13. https://doi.org/10. 1126/sciadv.aax3155

Dileep V, Gilbert DM (2018) Single-cell replication profiling to measure stochastic variation in mammalian replication timing. Nat Commun 9. https://doi.org/10.1038/s41467-017-02800-w

Ding D-Q, Okamasa K, Katou Y et al (2019) Chromosome-associated RNA-protein complexes promote pairing of homologous chromosomes during meiosis in Schizosaccharomyces pombe. Nat Commun 10:5598. https://doi.org/10.1038/s41467-019-13609-0

Ditlev JA, Case LB, Rosen MK (2018) Who's in and who's outcompositional control of biomolecular condensates. J Mol Biol 430:4666-4684. https://doi.org/10.1016/j.jmb.2018.08.003

Dora Tang TY, Rohaida Che Hak C, Thompson AJ et al (2014) Fatty acid membrane assembly on coacervate microdroplets as a step towards a hybrid protocell model. Nat Chem 6:527-533. https://doi.org/10. 1038/nchem.1921

Drobot B, Iglesias-Artola JM, Le Vay K et al (2018) Compartmentalised RNA catalysis in membrane-free coacervate protocells. Nat Commun 9:3643. https://doi.org/10.1038/s41467-018-06072-w

Erdel F, Rippe K (2018) Formation of chromatin subcompartments by phase separation. Biophys J 114:2262-2270. https://doi.org/10. 1016/j.bpj.2018.03.011

Falahati H, Pelham-Webb B, Blythe S, Wieschaus E (2016) Nucleation by rRNA dictates the precision of nucleolus assembly. Curr Biol 26: 277-285. https://doi.org/10.1016/j.cub.2015.11.065

Falk M, Feodorova Y, Naumova N et al (2019) Heterochromatin drives compartmentalization of inverted and conventional nuclei. Nature 570:395-399. https://doi.org/10.1038/s41586-019-1275-3

Forman-Kay JD, Mittag T (2013) From sequence and forces to structure, function, and evolution of intrinsically disordered proteins. Structure 21:1492-1499. https://doi.org/10.1016/j.str.2013.08.001

Forsythe JG, Yu S-S, Mamajanov I et al (2015) Ester-mediated amide bond formation driven by wet-dry cycles: a possible path to polypeptides on the prebiotic earth. Angew Chemie Int Ed 54:98719875. https://doi.org/10.1002/anie.201503792

Frankel EA, Bevilacqua PC, Keating CD (2016) Polyamine/nucleotide coacervates provide strong compartmentalization of $\mathrm{Mg} 2+$, nucleotides, and RNA. Langmuir 32:2041-2049. https://doi.org/10.1021/ acs.langmuir.5b04462

Freibaum BD, Taylor JP (2017) The role of dipeptide repeats in C9ORF72-related ALS-FTD. Front Mol Neurosci 10:1-9. https:// doi.org/10.3389/fnmol.2017.00035

Freibaum BD, Lu Y, Lopez-Gonzalez R et al (2015) GGGGCC repeat expansion in $\mathrm{C} 9$ orf72 compromises nucleocytoplasmic transport. Nature 525:129-133. https://doi.org/10.1038/nature14974

Fujioka Y, Alam JM, Noshiro D, Mouri K, Ando T, Okada Y, May AI, Knorr RL, Suzuki K, Ohsumi Y, Noda NN (2020) Phase separation organizes the site of autophagosome formation. Nature 578:301305. https://doi.org/10.1038/s41586-020-1977-6

Gibson BA, Doolittle LK, Schneider MWG et al (2019) Organization of chromatin by intrinsic and regulated phase separation. Cell 179: 470-484.e21. https://doi.org/10.1016/j.cell.2019.08.037

Gilbert N (2019) Biophysical regulation of local chromatin structure. Curr Opin Genet Dev 55:66-75. https://doi.org/10.1016/j.gde. 2019.06.001

Gillams R, Jia T (2018) Mineral surface-templated self-assembling systems: case studies from nanoscience and surface science towards origins of life research. Life 8:10. https://doi.org/10.3390/ life 8020010

Guenther EL, Cao Q, Trinh H et al (2018) Atomic structures of TDP-43 LCD segments and insights into reversible or pathogenic aggregation. Nat Struct Mol Biol 25:463-471. https://doi.org/10.1038/ s41594-018-0064-2

Guo L, Kim HJ, Wang H et al (2018) Nuclear-import receptors reverse aberrant phase transitions of RNA-binding proteins with prion-like domains. Cell 173:677-692.e20. https://doi.org/10.1016/j.cell.2018. 03.002

Guo YE, Manteiga JC, Henninger JE et al (2019) Transcriptional and splicing condensates. Nature. https://doi.org/10.1038/s41586-0191464-0

Guttenberg N, Virgo N, Chandru K et al (2017) Bulk measurements of messy chemistries are needed for a theory of the origins of life. Philos Trans R Soc A Math Phys Eng Sci 375:20160347. https:// doi.org/10.1098/rsta.2016.0347

Hansen JC (2002) Conformational dynamics of the chromatin fiber in solution: determinants, mechanisms, and functions. Annu Rev Biophys Biomol Struct 31:361-392. https://doi.org/10.1146/ annurev.biophys.31.101101.140858

Harrison AF, Shorter J (2017) RNA-binding proteins with prion-like domains in health and disease. Biochem J 474:1417-1438. https://doi. org/10.1042/BCJ20160499

Hentrich C, Szostak JW (2014) Controlled growth of filamentous fatty acid vesicles under flow. Langmuir 30:14916-14925. https://doi. org/10.1021/la503933x

Hirose T, Yamazaki T, Nakagawa S (2019) Molecular anatomy of the architectural NEAT1 noncoding RNA: the domains, interactors, and biogenesis pathway required to build phase-separated nuclear paraspeckles. Wiley Interdiscip Rev RNA 10:1-13. https://doi.org/ 10.1002/wrna. 1545

Hnisz D, Shrinivas K, Young RA et al (2017) A phase separation model for transcriptional control. Cell 169:13-23. https://doi.org/10.1016/ j.cell.2017.02.007

Hofweber M, Hutten S, Bourgeois B et al (2018) Phase separation of FUS is suppressed by its nuclear import receptor and arginine methylation. Cell 173:706-719.e13. https://doi.org/10.1016/j.cell.2018.03. 004

Hud NV, Milanovich FP, Balhorn R (1994) Evidence of novel secondary structure in DNA-bound protamine is revealed by Raman spectroscopy. Biochemistry 33:7528-7535. https://doi.org/10.1021/ bi00190a005

Iqbal M, Tao Y, Xie S et al (2016) Aqueous two-phase system (ATPS): an overview and advances in its applications. Biol Proced Online 18: 18. https://doi.org/10.1186/s12575-016-0048-8

Jain A, Vale RD (2017) RNA phase transitions in repeat expansion disorders. Nature 546:243-247. https://doi.org/10.1038/nature22386

Jeschke G (2012) DEER distance measurements on proteins. Annu Rev Phys Chem 63:419-446. https://doi.org/10.1146/annurevphyschem-032511-143716

Jia TZ, Hentrich C, Szostak JW (2014) Rapid RNA exchange in aqueous two-phase system and coacervate droplets. Orig Life Evol Biosph 44:1-12. https://doi.org/10.1007/s11084-014-9355-8

Jia TZ, Chandru K, Hongo Y et al (2019) Membraneless polyester microdroplets as primordial compartments at the origins of life. Proc Natl Acad Sci 116:15830-15835. https://doi.org/10.1073/ pnas.1902336116

Jordan SF, Rammu H, Zheludev IN et al (2019) Promotion of protocell self-assembly from mixed amphiphiles at the origin of life. Nat Ecol Evol 2019:31686020. https://doi.org/10.1038/s41559-019-1015-y

Karpen GH, Schaefer JE, Laird CD (1988) A Drosophila rRNA gene located in euchromatin is active in transcription and nucleolus formation. Genes Dev 2:1745-1763. https://doi.org/10.1101/gad.2. $12 b .1745$ 
Kato M, Han TW, Xie S et al (2012) Cell-free formation of RNA granules: low complexity sequence domains form dynamic fibers within hydrogels. Cell 149:753-767. https://doi.org/10.1016/j.cell.2012. 04.017

Keating CD (2012) Aqueous phase separation as a possible route to compartmentalization of biological molecules. Acc Chem Res 45: 2114-2124. https://doi.org/10.1021/ar200294y

Kedersha N, Anderson P (2009) Chapter 4 regulation of translation by stress granules and processing bodies. Prog Mol Biol Transl Sci 90: 155-185. https://doi.org/10.1016/S1877-1173(09)90004-7

Khrapunov SN, Dragan AI, Sivolob AV, Zagariya AM (1997) Mechanisms of stabilizing nucleosome structure. Study of dissociation of histone octamer from DNA. Biochim Biophys Acta Gene Struct Expr 1351:213-222. https://doi.org/10.1016/S0167-4781(96) 00199-6

Kilic S, Lezaja A, Gatti M et al (2019) Phase separation of 53 BP 1 determines liquid-like behavior of DNA repair compartments. EMBO J 38:1-17. https://doi.org/10.15252/embj.2018101379

Koga S, Williams DS, Perriman AW, Mann S (2011) Peptide-nucleotide microdroplets as a step towards a membrane-free protocell model. Nat Chem 3:720-724. https://doi.org/10.1038/nchem.1110

Kornberg RD, Lorch Y (1999) Twenty-five years of the nucleosome, fundamental particle of the eukaryote chromosome. Cell 98:285294. https://doi.org/10.1016/S0092-8674(00)81958-3

Kwon I, Kato M, Xiang S et al (2013) Phosphorylation-regulated binding of RNA polymerase II to fibrous polymers of low complexity domains. Cell 155:1049-1060. https://doi.org/10.1016/j.cell.2013.10. 033

Lachner M, O'Carroll D, Rea S et al (2001) Methylation of histone H3 lysine 9 creates a binding site for HP1 proteins. Nature 410:116 120. https://doi.org/10.1038/35065132

Larson AG, Elnatan D, Keenen MM et al (2017) Liquid droplet formation by HP1 $\alpha$ suggests a role for phase separation in heterochromatin. Nature 547:236-240. https://doi.org/10.1038/nature22822

Li P, Banjade S, Cheng HC et al (2012) Phase transitions in the assembly of multivalent signalling proteins. Nature 483:336-340. https://doi. org/10.1038/nature10879

Li L, Prywes N, Tam CP et al (2017) Enhanced nonenzymatic RNA copying with 2-aminoimidazole activated nucleotides. J Am Chem Soc 139:1810-1813. https://doi.org/10.1021/jacs.6b13148

Lieberman-Aiden E, van Berkum NL, Williams L et al (2009) Comprehensive mapping of long-range interactions reveals folding principles of the human genome. Science 326(80):289-293. https:// doi.org/10.1126/science.1181369

Lin Y, Mori E, Kato M et al (2016) Toxic PR poly-dipeptides encoded by the $\mathrm{C} 9$ orf72 repeat expansion target LC domain polymers. Cell 167: 789-802.e12. https://doi.org/10.1016/j.cell.2016.10.003

Luisi PL, Walde P, Oberholzer T (1999) Lipid vesicles as possible intermediates in the origin of life. Curr Opin Colloid Interface Sci 4:3339. https://doi.org/10.1016/S1359-0294(99)00012-6

Luo F, Gui X, Zhou H et al (2018) Atomic structures of FUS LC domain segments reveal bases for reversible amyloid fibril formation. Nat Struct Mol Biol 25:341-346. https://doi.org/10.1038/s41594-0180050-8

Maeshima K, Imai R, Tamura S, Nozaki T (2014) Chromatin as dynamic 10-nm fibers. Chromosoma 123:225-237. https://doi.org/10.1007/ s00412-014-0460-2

Maharana S, Wang J, Papadopoulos DK et al (2018) RNA buffers the phase separation behavior of prion-like RNA binding proteins. Science 360(80):918-921. https://doi.org/10.1126/science.aar7366

Mainz A, Religa TL, Sprangers R et al (2013) NMR spectroscopy of soluble protein complexes at one mega-dalton and beyond. Angew Chemie Int Ed 52:8746-8751. https://doi.org/10.1002/anie. 201301215
Mann S (2012) Systems of creation: the emergence of life from nonliving matter. Acc Chem Res 45:2131-2141. https://doi.org/10.1021/ ar200281t

Mansy SS, Szostak JW (2009) Reconstructing the emergence of cellular life through the synthesis of model protocells. Cold Spring Harb Symp Quant Biol 74:47-54. https://doi.org/10.1101/sqb.2009.74. 014

Marquet P, Depeursinge C, Magistretti PJ (2013) Exploring neural cell dynamics with digital holographic microscopy. Annu Rev Biomed Eng 15:407-431. https://doi.org/10.1146/annurev-bioeng-071812152356

Martin N, Li M, Mann S (2016) Selective uptake and refolding of globular proteins in coacervate microdroplets. Langmuir 32:5881-5889. https://doi.org/10.1021/acs.langmuir.6b01271

Martin N, Tian L, Spencer D et al (2019) Photoswitchable phase separation and oligonucleotide trafficking in DNA coacervate microdroplets. Angew Chemie Int Ed 58:14594-14598. https:// doi.org/10.1002/anie.201909228

Masui O, Bonnet I, Le Baccon P et al (2011) Live-cell chromosome dynamics and outcome of $\mathrm{X}$ chromosome pairing events during ES cell differentiation. Cell 145:447-458. https://doi.org/10.1016/j. cell.2011.03.032

Mateju D, Franzmann TM, Patel A et al (2017) An aberrant phase transition of stress granules triggered by misfolded protein and prevented by chaperone function. EMBO J 36:1669-1687. https:// doi.org/10.15252/embj.201695957

Matsumura S, Kun A, Ryckelynck M et al (2016) Transient compartmentalization of RNA replicators prevents extinction due to parasites. Science 354(80):1293-1296. https://doi.org/10.1126/science. aag 1582

Milshteyn D, Damer B, Havig J, Deamer D (2018) Amphiphilic compounds assemble into membranous vesicles in hydrothermal hot spring water but not in seawater. Life 8:11. https://doi.org/10.3390/ life 8020011

Montanaro L, Treré D, Derenzini M (2008) Nucleolus, ribosomes, and cancer. Am J Pathol 173:301-310. https://doi.org/10.2353/ajpath. 2008.070752

Monterroso B, Zorrilla S, Sobrinos-Sanguino M et al (2016) Microenvironments created by liquid-liquid phase transition control the dynamic distribution of bacterial division FtsZ protein. Sci Rep 6:1-13. https://doi.org/10.1038/srep35140

Monterroso B, Zorrilla S, Sobrinos-Sanguino M et al (2019) Bacterial FtsZ protein forms phase-separated condensates with its nucleoidassociated inhibitor SlmA. EMBO Rep 20:1-13. https://doi.org/10. 15252/embr.201845946

Mori K, Weng S-M, Arzberger T et al (2013) The C9orf72 GGGGCC repeat is translated into aggregating dipeptide-repeat proteins in FTLD/ALS. Science 339(80):1335-1338. https://doi.org/10.1126/ science. 1232927

Murray DT, Tycko R (2020) Sidechain hydrogen bonding interactions within amyloid-like fibrils formed by the low-complexity domain of FUS: evidence from solid state nuclear magnetic resonance spectroscopy. Biochemistry. https://doi.org/10.1021/acs.biochem. $9 \mathrm{~b} 00892$

Murray DT, Kato M, Lin Y et al (2017) Structure of FUS protein fibrils and its relevance to self-assembly and phase separation of lowcomplexity domains. Cell 171:615-627.e16. https://doi.org/10. 1016/j.cell.2017.08.048

Murthy AC, Dignon GL, Kan Y et al (2019) Molecular interactions underlying liquid-liquid phase separation of the FUS low-complexity domain. Nat Struct Mol Biol 26:637-648. https://doi.org/10.1038/ s41594-019-0250-x

Nannenga BL, Gonen T (2019) The cryo-EM method microcrystal electron diffraction (MicroED). Nat Methods 16:369-379. https://doi. org/10.1038/s41592-019-0395-X 
Nott TJ, Petsalaki E, Farber P et al (2015) Phase transition of a disordered nuage protein generates environmentally responsive membraneless organelles. Mol Cell 57:936-947. https://doi.org/10.1016/j.molcel. 2015.01.013

Nozaki T, Imai R, Tanbo M et al (2017) Dynamic organization of chromatin domains revealed by super-resolution live-cell imaging. Mol Cell 67:282-293.e7. https://doi.org/10.1016/j.molcel.2017.06.018

Nozawa RS, Gilbert N (2019) RNA: nuclear glue for folding the genome. Trends Cell Biol 29:201-211. https://doi.org/10.1016/j.tcb.2018.12. 003

Nozawa RS, Nagao K, Masuda HT et al (2010) Human POGZ modulates dissociation of HP1 $\alpha$ from mitotic chromosome arms through Aurora B activation. Nat Cell Biol 12:719-727. https://doi.org/10. 1038/ncb2075

Nozawa RS, Boteva L, Soares DC et al (2017) SAF-A regulates interphase chromosome structure through Oligomerization with chromatin-associated RNAs. Cell 169:1214-1227.e18. https://doi. org/10.1016/j.cell.2017.05.029

Oakes ML, Johzuka K, Vu L et al (2006) Expression of rRNA genes and nucleolus formation at ectopic chromosomal sites in the yeast Saccharomyces cerevisiae. Mol Cell Biol 26:6223-6238. https:// doi.org/10.1128/mcb.02324-05

Pagès G, Gilard V, Martino R, Malet-Martino M (2017) Pulsed-field gradient nuclear magnetic resonance measurements (PFG NMR) for diffusion ordered spectroscopy (DOSY) mapping. Analyst 142: 3771-3796. https://doi.org/10.1039/C7AN01031A

Parker ET, Cleaves HJ, Bada JL, Fernández FM (2016) Quantitation of $\alpha$ hydroxy acids in complex prebiotic mixtures via liquid chromatography/tandem mass spectrometry. Rapid Commun Mass Spectrom 30:2043-2051. https://doi.org/10.1002/rcm.7684

Parker MW, Bell M, Mir M et al (2019) A new class of disordered elements controls DNA replication through initiator self-assembly. Elife 8:1-35. https://doi.org/10.7554/eLife.48562

Pascal R, Boiteau L (2011) Energy flows, metabolism and translation. Philos Trans R Soc B Biol Sci 366:2949-2958. https://doi.org/10. 1098/rstb.2011.0135

Patel A, Lee HO, Jawerth L et al (2015) A liquid-to-solid phase transition of the ALS protein FUS accelerated by disease mutation. Cell 162: 1066-1077. https://doi.org/10.1016/j.cell.2015.07.047

Peltzer ET, Bada JL (1978) $\alpha$-Hydroxycarboxylic acids in the Murchison meteorite. Nature 272:443-444. https://doi.org/10.1038/272443a0

Pir Cakmak F, Keating CD (2017) Combining catalytic microparticles with droplets formed by phase coexistence: adsorption and activity of natural clays at the aqueous/aqueous Interface. Sci Rep 7:1-14. https://doi.org/10.1038/s41598-017-03033-Z

Poudyal RR, Pir Cakmak F, Keating CD, Bevilacqua PC (2018) Physical principles and extant biology reveal roles for RNA-containing membraneless compartments in origins of life chemistry. Biochemistry 57:2509-2519. https://doi.org/10.1021/acs.biochem. $8 \mathrm{~b} 00081$

Poudyal RR, Guth-Metzler RM, Veenis AJ et al (2019) Template-directed RNA polymerization and enhanced ribozyme catalysis inside membraneless compartments formed by coacervates. Nat Commun 10:490. https://doi.org/10.1038/s41467-019-08353-4

Priftis D, Tirrell M (2012) Phase behaviour and complex coacervation of aqueous polypeptide solutions. Soft Matter 8:9396-9405. https:// doi.org/10.1039/C2SM25604E

Priftis D, Megley K, Laugel N, Tirrell M (2013) Complex coacervation of poly (ethylene-imine)/polypeptide aqueous solutions: thermodynamic and rheological characterization. J Colloid Interface Sci 398:39-50. https://doi.org/10.1016/j.jcis.2013.01.055

Qamar S, Wang GZ, Randle SJ et al (2018) FUS phase separation is modulated by a molecular chaperone and methylation of arginine cation- $\pi$ interactions. Cell 173:720-734. https://doi.org/10.1016/j. cell.2018.03.056
Qiao Y, Li M, Booth R, Mann S (2017) Predatory behaviour in synthetic protocell communities. Nat Chem 9:110-119. https://doi.org/10. 1038/nchem. 2617

Rai AK, Chen J-X, Selbach M, Pelkmans L (2018) Kinase-controlled phase transition of membraneless organelles in mitosis. Nature 559:211-216. https://doi.org/10.1038/s41586-018-0279-8

Ryan VH, Dignon GL, Zerze GH et al (2018) Mechanistic view of hnRNPA2 low-complexity domain structure, interactions, and phase separation altered by mutation and arginine methylation. Mol Cell 69:465-479.e7. https://doi.org/10.1016/j.molcel.2017.12.022

Sabari BR, Dall'Agnese A, Boija A et al (2018) Coactivator condensation at super-enhancers links phase separation and gene control. Science 361(80):eaar3958. https://doi.org/10.1126/science.aar3958

Saio T, Ishimori K (2019) Accelerating structural life science by paramagnetic lanthanide probe methods. Biochim Biophys Acta Gen Subj 1864:129332. https://doi.org/10.1016/j.bbagen.2019.03.018

Saio T, Guan X, Rossi P et al (2014) Structural basis for protein antiaggregation activity of the trigger factor chaperone. Science 344(80):1250494. https://doi.org/10.1126/science.1250494

Sakamoto K, Katayama R, Asaka R et al (2018) Recurrent 8q24 rearrangement in blastic plasmacytoid dendritic cell neoplasm: association with immunoblastoid cytomorphology, MYC expression, and drug response. Leukemia 32:2590-2603. https://doi.org/10.1038/ s41375-018-0154-5

Sanulli S, Trnka MJ, Dharmarajan V et al (2019) HP1 reshapes nucleosome core to promote phase separation of heterochromatin. Nature 575:390-394. https://doi.org/10.1038/s41586-019-1669-2

Schiessel H (2003) The physics of chromatin. J Phys Condens Matter 15: R699-R774. https://doi.org/10.1088/0953-8984/15/19/203

Schmidt HB, Görlich D (2016) Transport selectivity of nuclear pores, phase separation, and membraneless organelles. Trends Biochem Sci 41:46-61. https://doi.org/10.1016/j.tibs.2015.11.001

Shi KY, Mori E, Nizami ZF et al (2017) Toxic PR n poly-dipeptides encoded by the C9orf72 repeat expansion block nuclear import and export. Proc Natl Acad Sci 114:E1111-E1117. https://doi.org/ $10.1073 /$ pnas. 1620293114

Shin Y, Brangwynne CP (2017) Liquid phase condensation in cell physiology and disease. Science 357(80):eaaf4382. https://doi.org/10. 1126/science.aaf4382

Shin Y, Chang YC, Lee DSW et al (2018) Liquid nuclear condensates mechanically sense and restructure the genome. Cell 175:14811491.e13. https://doi.org/10.1016/j.cell.2018.10.057

Shirt-Ediss B, Murillo-Sánchez S, Ruiz-Mirazo K (2017) Framing major prebiotic transitions as stages of protocell development: three challenges for origins-of-life research. Beilstein J Org Chem 13:1388 1395. https://doi.org/10.3762/bjoc.13.135

Smith E, Morowitz H (2016) The origin and nature of life on earth: the emergence of the fourth geosphere. Cambridge, Cambridge University Press. https://doi.org/10.1017/CBO9781316348772

Soniat M, Chook YM (2015) Nuclear localization signals for four distinct Karyopherin- $\beta$ nuclear import systems. Biochem J 468:353-362. https://doi.org/10.1042/BJ20150368

Strom AR, Emelyanov AV, Mir M et al (2017) Phase separation drives heterochromatin domain formation. Nature 547:241-245. https:// doi.org/10.1038/nature22989

Szostak JW, Bartel DP, Luisi PL (2001) Synthesizing life. Nature 409: 387-390. https://doi.org/10.1038/35053176

Takahashi S, Miura H, Shibata T et al (2019) Genome-wide stability of the DNA replication program in single mammalian cells. Nat Genet 51:529-540. https://doi.org/10.1038/s41588-019-0347-5

Taniguchi S, Watanabe N, Nose T, Maeda I (2016) Development of short and highly potent self-assembling elastin-derived pentapeptide repeats containing aromatic amino acid residues. J Pept Sci 22:36-42. https://doi.org/10.1002/psc.2837

Trivedi P, Palomba F, Niedzialkowska E et al (2019) The inner centromere is a biomolecular condensate scaffolded by the chromosomal 
passenger complex. Nat Cell Biol 21:1127-1137. https://doi.org/10. 1038/s41556-019-0376-4

Tsang B, Arsenault J, Vernon RM et al (2019) Phosphoregulated FMRP phase separation models activity-dependent translation through bidirectional control of mRNA granule formation. Proc Natl Acad Sci U S A 116:4218-4227. https://doi.org/10.1073/pnas.1814385116

Turner AL, Watson M, Wilkins OG et al (2018) Highly disordered histone H1-DNA model complexes and their condensates. Proc Natl Acad Sci U S A 115:11964-11969. https://doi.org/10.1073/pnas. 1805943115

Veis A (2011) A review of the early development of the thermodynamics of the complex coacervation phase separation. Adv Colloid Interf Sci 167:2-11. https://doi.org/10.1016/j.cis.2011.01.007

Walker SI, Packard N, Cody GD (2017) Re-conceptualizing the origins of life introduction. Philos Trans R Soc A Math Phys Eng Sci 375: 20160337

Wang R, Yang X, Cui L et al (2019) Gels of amyloid fibers. Biomolecules 9:210. https://doi.org/10.3390/biom9060210

Weber SC, Brangwynne CP (2015) Inverse size scaling of the nucleolus by a concentration-dependent phase transition. Curr Biol 25:641646. https://doi.org/10.1016/j.cub.2015.01.012

Widom J (1998) Chromatin structure: linking structure to function with histone H1. Curr Biol 8:788-791. https://doi.org/10.1016/s09609822(07)00500-3

Xiang S, Kato M, Wu LC et al (2015) The LC domain of hnRNPA2 adopts similar conformations in hydrogel polymers, liquid-like droplets, and nuclei. Cell 163:829-839. https://doi.org/10.1016/j. cell.2015.10.040
Yanagisawa M, Yamashita Y, Mukai S et al (2014) Phase separation in binary polymer solution: gelatin/poly (ethylene glycol) system. J Mol Liq 200:2-6. https://doi.org/10.1016/j.molliq.2013.12.035

Yang Y, Fang Z, Chen X et al (2017) An overview of pickering emulsions: solid-particle materials, classification, morphology, and applications. Front Pharmacol 8:1-20. https://doi.org/10.3389/fphar. 2017.00287

Yin Y, Niu L, Zhu X et al (2016) Non-equilibrium behaviour in coacervate-based protocells under electric-field-induced excitation. Nat Commun 7:10658. https://doi.org/10.1038/ncomms 10658

Yoshizawa T, Matsumura H (2020) Effect of nuclear import receptors on liquid-liquid phase separation. Biophys Physicobiol 12:103-117. https://doi.org/10.2142/biophysico.BSJ-2019052

Yoshizawa T, Ali R, Jiou J et al (2018) Nuclear import receptor inhibits phase separation of FUS through binding to multiple sites. Cell 173: 693-705. https://doi.org/10.1016/j.cell.2018.03.003

Zhang K, Daigle JG, Cunningham KM et al (2018) Stress granule assembly disrupts nucleocytoplasmic transport. Cell 173:958-971.e17. https://doi.org/10.1016/j.cell.2018.03.025

Zhu TF, Szostak JW (2009) Coupled growth and division of model protocell membranes. J Am Chem Soc 131:5705-5713. https://doi. org/10.1021/ja900919c

Zhu TF, Adamala K, Zhang N, Szostak JW (2012) Photochemically driven redox chemistry induces protocell membrane pearling and division. Proc Natl Acad Sci U S A 109:9828-9832. https://doi. org/10.1073/pnas.1203212109

Publisher's note Springer Nature remains neutral with regard to jurisdictional claims in published maps and institutional affiliations. 